\title{
A Declarative Approach for First-Order Built-in's of Prolog ${ }^{\star}$
}

\author{
Krzysztof R. Apt ${ }^{1}$, Elena Marchiori ${ }^{2}$, Catuscia Palamidessi ${ }^{3}$ \\ ${ }^{1}$ CWI, P.O. Box 94079, NL-1090 GB Amsterdam, The Netherlands and Faculty of Mathematics \\ and Computer Science, University of Amsterdam, Plantage Muidergracht 24 NL-1018 TV \\ Amsterdam, The Netherlands \\ ${ }^{2}$ CWI, P.O. Box 94079, NL-1090 GB Amsterdam, The Netherlands \\ ${ }^{3}$ Dipartimento di Informatica e Scienze dell' Informazione, Università di Genova, Viale Benedetto \\ XV 3, I-16132 Genova, Italy
}

Received November 9, 1992; revised version April 19, 1993

\begin{abstract}
We provide here a framework for studying Prolog programs with various built-in's that include arithmetic operations, and such metalogical relations like var and ground. To this end we propose a new, declarative semantics and prove completeness of the Prolog computation mechanism w.r.t. this semantics. We also show that this semantics is fully abstract in an appropriate sense. Finally, we provide a method for proving termination of Prolog programs with built-in's which uses this semantics. The method is shown to be modular and is illustrated by proving termination of a number of programs including the unify program of Sterling and Shapiro [17].
\end{abstract}

Keywords: Prolog programs, Built-in's, Declarative semantics, Termination.

\section{Introduction}

\subsection{Motivation}

Theory of logic programming allows us to treat formally only pure Prolog programs, that is those whose syntax is based on Horn clauses. Any formal treatment of more realistic Prolog programs has to take into account the use of various built-in's. Some of them, like arithmetic relations, seem to be trivial to handle, as they simply refer to some theory of arithmetic. However, the restrictions on the form of their arguments (like the requirement that both arguments of $<$ should be ground) cause complications which the theory of logic programming does not properly account

* 1991 Mathematics Subject Classification: 68Q40, 68T15,

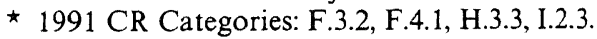


for. In particular, in presence of arithmetic relations the independence of the refutability from the selection rule fails, as the goal $\leftarrow x=2,1<x$ shows.

Further, the use of metalogical relations (like var, ground) leads to various additional problems. Clearly, var cannot be handled using the traditional semantics based on first-order logic because $\operatorname{var}(x)$ is true whereas some instances of it are not. In presence of nonvar another complication arises: the well-known Lifting Lemma (see Lloyd [14]) used to prove completeness of the SLD-resolution does not hold - for a non-variable term $t$ the goal $\leftarrow$ nonvar $(t)$ can be refuted whereas its more general version $\leftarrow$ nonvar $(x)$ cannot.

Finally, study of termination of Prolog programs in presence of the above built-in's calls for some new insights. For example, the program list

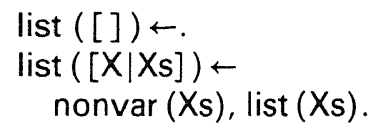

which recognizes a list, always terminates, whereas its pure Prolog counterpart obtained by dropping the atom nonvar $(X s)$ may diverge.

The aim of this paper is to provide a systematic account of the class of the above mentioned built-in's of Prolog. This class includes the arithmetic relations (like +, $<$ etc.) and some metalogical relations (like var, ground etc.). To distinguish them from those built-in's which refer to clauses and goals (like call and assert), we call them first-order built-in's. Hence the title.

The main tool in our approach is a new, non-standard declarative semantics which associates with each relation symbol input and output substitutions. It is introduced in Sect. 2. We also prove there a completeness result connecting this semantics with the Prolog computational mechanism. We show that this semantics is a natural extension of the S-semantics by Falaschi et al. [12], in the sense that it is isomorphic to the $S$-semantics for pure Prolog programs. Moreover we show that our semantics is in a sense the most simple extension, by proving that it is fully abstract w.r.t. goals conjunctions.

This semantics is crucial for the study of termination of Prolog programs that use the first-order built-in's. Our approach to this subject combines the use of the level mapping functions (that assign elements from a well-founded set to atoms) with the above semantics. In this respect it is thus similar to that of Apt and Pedreschi [5] which called for the use of level mappings assigning natural numbers to ground atoms, and declarative semantics. However, important differences arise due to the presence of built-in's. First, we have to analyze the original program and not its ground version. Second, in presence of first-order built-in's it seems natural to study programs that terminate for all goals and not only for all ground goals as in Apt and Pedreschi [5]. So different characterization results are needed. These issues are dealt with in Sect. 3 where we also show how termination of Prolog programs with first-order built-in's can be dealt with in a modular way.

In Sect. 4 we apply our approach to termination to prove termination of the above list program, the typed version of the append program and a version of the unify program of Sterling and Shapiro [17].

We are aware of two other approaches to define the meaning of Prolog firstorder built-in's, namely that of Börger [7] based on so-called dynamic algebras, and that of Deransart and Ferrand [11] based on an abstract interpreter.

Their aim is to provide semantics to the complete Prolog language whereas ours 
is to extend the declarative semantics to Prolog programs with first-order built-in's so that one can reason about such programs. In this respect our approach has the same aim as that of Hill and Lloyd [13] where all metalogical features of Prolog are represented in a uniform way by means of a representation of the object level in the meta-level, reminiscent of the Gödelization process in Peano arithmetic.

\subsection{Preliminaries}

In what follows we study logic programs extended by various built-in relations. We call the resulting objects Prolog programs, or simply programs, and identify pure Prolog programs with logic programs. Prolog programs can be executed by means of the LD-resolution, which consists of the usual SLD-resolution combined with the leftmost selection rule, that is appropriately extended to deal with the built-in relations. By length $l(\xi)$ of an LD-derivation $\xi$ we mean the number of its goals.

Given an expression (term, atom, goal,...) or a substitution $E$ we denote the set of variables occurring in it by $\operatorname{Var}(E)$. We often write $\eta \mid E$ to denote $\eta \mid \operatorname{Var}(E)$. The set of all variables is denoted by $V a r$. We often manipulate various sets of variables. In general $\mathbf{x}, \mathbf{y}$ stands for sequences of different variables. Sometimes we identify such sequences with sets of variables. Given a substitution $\eta$ and a set of variables $\mathbf{x}$ we denote by $\eta \mid \mathbf{x}$ the substitution obtained from $\eta$ by restricting its domain, $\operatorname{Dom}(\eta)$, to $\mathbf{x}$. By $\operatorname{Ran}(\eta)$ we denote the set of variables that appear in the terms of the range of $\eta$. A renaming is a substitution that is a permutation of the variables constituting its domain. We use mgu as a shorthand for most general unifier.

Recall that an mu $\eta$ of $A$ and $B$ is idempotent if $\eta \eta=\eta$ and is relevant if $\operatorname{Ran}(\eta) \subseteq \operatorname{Var}(A, B)$. The relation more general than defined on pairs of atoms, terms or substitutions is denoted by $\leqq$.

Let $s$ be a term. Then $s_{i}$ denotes the $i$-th argument of $s$, when it is defined, nodes $(s)$ denotes the number of nodes of $s$ in the tree representation, $a(s)$ denotes the arity of the principal functor of $s$ and funct (s) denotes its function symbol.

It is convenient to associate with each pair of terms that unify a unique idempotent (hence relevant) mgu in the sense of Apt [1] [p. 502]. Given such a pair $s, t$ we denote it by $m g u(s, t)$. Further, we associate with each pair of sequences of terms that unify a unique idempotent (hence relevant) mgu defined as follows.

- $\operatorname{mgu}((),())=\varepsilon$, where () indicates the empty sequence;

- $\operatorname{mgu}((s, \mathbf{s}),(t, \mathbf{t}))=\alpha \operatorname{mgu}((\mathbf{s} \alpha),(\mathbf{t} \alpha))$, where $\alpha=\operatorname{mgu}(s, t)$.

We write $m g u(\mathbf{s}, \mathbf{t})$ instead of $m g u((\mathbf{s}),(\mathbf{t}))$. It is not difficult to show that $m g u(\mathbf{s}, \mathbf{t})$ is indeed an idempotent mgu of $\mathbf{s}$ and $\mathbf{t}$. Then we associate with each pair of atoms $A$ and $B$ that unify $m g u(\mathbf{s}, \mathbf{t})$, where $\mathbf{s}$ and $\mathbf{t}$ are the sequences of arguments respectively of $A$ and $B$. We denote this $m g u$ by $m g u(A, B)$.

Atoms of the form $p(\mathbf{x})$ where $p$ is a relation are called elementary atoms and atoms containing a built-in relation are referred to as built-in atoms. Finally, atoms containing a relation used in a head of a clause of a program $P$ are said to be defined in $P$. In the context of logic programs, or more generally, Prolog programs, it is convenient to treat sequences of atoms as conjunctions (sometimes called conjuncts). Usually A, B denote such conjuncts.

The rest of the used notation is more or less standard and essentially follows Lloyd [14]. Recall that, if $\theta_{1}, \ldots, \theta_{n}$ are the consecutive mgu's along a refutation of 
a goal $G$ in the program $P$, then the restriction $\left(\theta_{1} \ldots \theta_{n}\right) \mid \operatorname{Var}(G)$ of $\theta_{1} \ldots \theta_{n}$ to the variables of $G$ is called computed answer substitution (c.a.s. for short) of $P \cup\{G\}$. In this paper we also associate c.a.s.'s with prefixes of LD-derivations in the obvious way. These prefixes of LD-derivations are also called partial derivations.

\section{The Declarative Semantics}

\subsection{Motivation}

In this section we define a declarative semantics appropriate to describe the operational behaviour of Prolog programs. First, let us see why it is impossible to achieve this goal by simply modifying one of the usually considered declarative semantics.

The standard declarative semantics, based on the (ground) Herbrand models due to van Emden and Kowalski [18], is clearly inadequate to deal with first-order built-in's. Indeed, in this semantics in a given interpretation if an atom is true then all its ground instances are. However, for every ground term $t, \operatorname{var}(t)$ should be false in every model whereas $\operatorname{var}(x)$ should be true. Therefore we say that var is a non-monotonic relation.

We conclude that any declarative modeling of non-monotonic relations requires an explicit introduction of non-ground atoms in the Herbtand interpretations, in order to define the truth value of an atom independently from its ground instances. The first declarative semantics based on non-ground atoms was given by Clark $[10]$, with the aim of defining the validity of open atoms (like $p(x)$ ) in terms of their truth value in the least Herbrand model. Successively, other declarative models based on non-ground atoms were investigated in Falaschi et al. [12]: the C-semantics which was shown to be equivalent to Clark's semantics, and the S-semantics. However, all these models are not suitable for Prolog programs, because - like the standard semantics of van Emden and Kowalski [18], the resulting definition of truth treats the body of a clause as a logical conjunction - i.e. the ", is interpreted as an 'and', and this means that the order of the literals in the body is irrelevant. On the other hand, the presence of built-in relations - in particular of the nonmonotonic ones, makes this order relevant. Consider for instance

$$
\begin{aligned}
P_{1}: & p(X) \leftarrow \operatorname{var}(X), q(X) . \\
& q(a) \leftarrow .
\end{aligned}
$$

and

$$
\begin{aligned}
P_{2}: & p(X) \leftarrow q(X), \operatorname{var}(X) . \\
& q(a) \leftarrow .
\end{aligned}
$$

The behavior of the goal $\leftarrow p(x)$ in these programs is different (in $P_{1}$ it succeeds, whereas in $P_{2}$ it fails). In other words, the independence from the selection rule, and the Switching Lemma of Lloyd [14] do not hold for Prolog programs. If we want to characterize declaratively the operational behaviour of goals, we must therefore describe the meaning of ', in the body of clauses in a non-commutative way, more precisely, we have to mimic the leftmost selection rule of Prolog.

However, the intended model cannot be obtained simply by modifying the interpretation of ', in the C-semantics. The reason is that the domain structure of the C-semantics is too poor: it does not allow us to model the meaning of non- 
monotonic relations. Indeed, in the $\mathrm{C}$-semantics the interpretations are upward closed, that is, if $A$ belongs to (is true in) an interpretation $I$, then all its instances belong to $I$, as well.

On the other hand, in the S-semantics the interpretations are not upward closed. However, the $\mathrm{S}$-semantics is monotonic, that is $A$ is true in an interpretation $I$ if a more general version of $A$ belongs to $I$.

Moreover, in presence of built-in relations like nonvar, another problem arises: the goal $\leftarrow$ nonvar $(x)$ fails whereas for every non-variable term $t$ the goal $\leftarrow$ nonvar $(t)$ succeeds. Therefore we say that nonvar is a non-down-monotonic relation. Due to the presence of non-down-monotonic relations the Lifting Lemma (see Lloyd [14]) does not hold for Prolog programs. Consider for instance

$P_{3}: p(X) \leftarrow$ nonvar $(X)$.

With this program for every non-variable term $t$, the goal $\leftarrow p(t)$ has a refutation, whereas $\leftarrow p(x)$ fails.

This example shows that it is not sufficient to identify the meaning of a relation $p$ with the set of (computed answer) substitutions $\eta$ which $p$ is able to compute - in a sense, the post-conditions which are verified after the possible executions of the goal $\leftarrow p(\mathbf{x})$. We also need a pre-condition, i.e. information about the substitution $\theta$ by which the atom $p(\mathbf{x})$ is instantiated before starting the computation. A possible way to do it is by enriching the domain with another component, thus explicitly representing the substitution before execution.

\section{$2.2 \Theta$-Semantics}

This leads us to consider objects of the form $\langle\theta, p(\mathbf{x}), \eta\rangle$, where $\theta$ represents the pre-substitution (or input substitution) and $\eta$ represents the post-substitution (or output substitution) for the goal $\leftarrow p(\mathbf{x})$. For technical convenience we equivalently represent these triples as pairs of the form $\langle A, \eta\rangle$, where $A$ is the atom obtained by the application of the input substitution $\theta$ to the elementary atom $p(\mathbf{x})$, i.e. $A=p(\mathbf{x}) \theta$. In Sect. 2.6 we prove the full abstraction of this model, thus showing that all the information we encode in this semantical structure is in fact necessary.

Of course, we can restrict our attention to pairs $\langle A, \eta\rangle$ in which $\eta$ does not affect the variables that do not appear in $A$.

First, we deal with built-in relations. For any such relation $p$ we stipulate a set $\llbracket p \rrbracket$ of pairs defining its operational behaviour. We list here some cases. In the definition below, "=" is the well-known built-in standing for "is unifiable with".

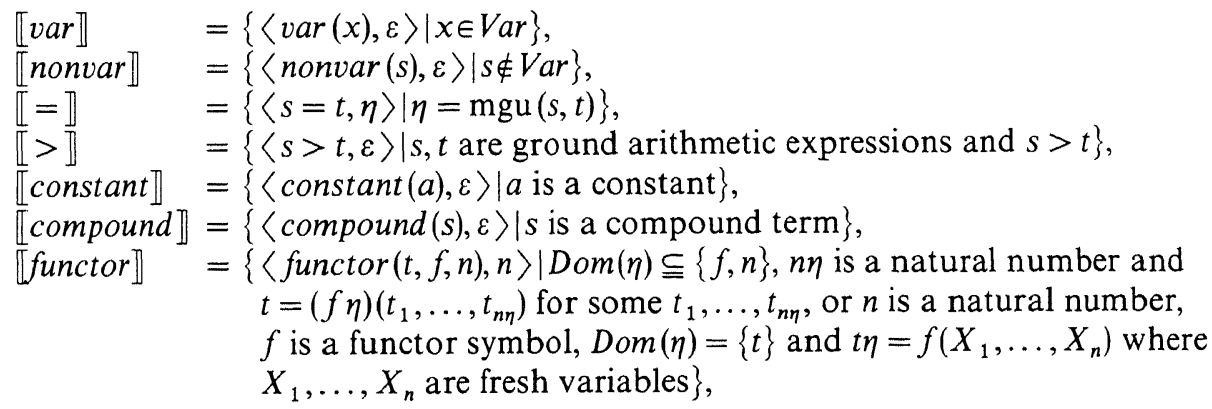




$$
\begin{aligned}
& \mathbb{I}:=\mathbb{I} \quad=\{\langle x:=s,\{x / t\}\rangle \mid x \in \text { Var, } s \text { is a ground arithmetic expression with } \\
& \text { value } t\} \text {, } \\
& {[\arg ]=\left\{\langle\arg (n, s, t), \eta\rangle \mid \operatorname{Dom}(\eta) \subseteq\{t\} \text { and } t \eta=s_{n} \text { or } \operatorname{Dom}(\eta)=\left\{s_{n}\right\}\right. \text { and }} \\
& \left.s_{n} \eta=t\right\} \text {, } \\
& ==\mathbb{Z}=\{\langle s\rangle==t, \varepsilon\rangle \mid s \neq t\} \text {. }
\end{aligned}
$$

We assume that the set of pairs associated with a built-in relation describes correctly its operational behaviour, in the following sense.

Definition 2.1. Let $A$ be an atom with a built-in relation $p$. Then for every conjunct $\mathbf{B}$ the goal $\leftarrow \mathbf{B} \eta$ is a resolvent of $\leftarrow A, \mathbf{B}$ iff $\langle A, \eta\rangle \in \llbracket p \rrbracket$.

Notice that in our approach we do not distinguish between failures and errors. For example, in Prolog the evaluation of the goal $\leftarrow X:=Y+1$ will result in an error and not in a (back-trackable) failure. By further refining the structure of the sets $[p]$ we could easily incorporate this distinction in the semantics.

We consider now atoms defined by the program. First we introduce the following generalization of Herbrand base and Herbrand interpretation.

\section{Definition 2.2. ( $\Theta$-domain and $\Theta$-interpretation)}

- Let $P$ be a Prolog program. The $\Theta$-base $\Theta_{P}$ of $P$ is the set of all pairs $\langle A, \eta\rangle$, where $A$ is an atom defined in $P$, and $\eta$ is a substitution s.t. $\operatorname{Dom}(\eta) \subseteq \operatorname{Var}(A)$.

- A $\Theta$-interpretation $\mathscr{I}$ of $P$ is a subset of the $\Theta$-base $\Theta_{P}$.

To define the truth in $\Theta$-interpretations we have to model appropriately the proof theoretic properties of the computed answer substitutions. To this end it is important to reflect on them first.

The following lemma relates c.a.s.'s of resolvents of a goal with c.a.s.'s of the goal. It is a consequence of Corollary 3.5 of Apt and Doets [3], to which the reader is referred for the proof.

Lemma 2.3 (C.a.s.) Consider an atomic goal $\leftarrow A$ with input clause $p(\mathbf{x}) \leftarrow \mathbf{B}$. Let $\theta=m g u(A, p(\mathbf{x}))$ be s.t. $\operatorname{Dom}(\theta)=\mathbf{x}$ and let $\eta$ be a c.a.s. of $P \cup\{\leftarrow \mathbf{B} \theta\}$. Suppose that $\operatorname{Ran}(\eta) \cap \operatorname{Var}(p(\mathbf{x}) \theta) \cong \operatorname{Var}(\mathbf{B} \theta)$. Then $\eta \mid A$ is a c.a.s. of $P \cup\{\leftarrow A\}$.

This Lemma provides a sufficient condition to guarantee that a c.a.s. of a goal coincides with a c.a.s. of its resolvent on the variables of the goal. Let us give an example showing that this condition is needed. Consider the program $P$ :

$$
\begin{aligned}
& p(X, Y) \leftarrow q(X) . \\
& q(X) \leftarrow X=f(Y) .
\end{aligned}
$$

and the goal $\leftarrow p(X, Y)$. Take as input clause $p\left(X^{\prime}, Y^{\prime}\right) \leftarrow q\left(X^{\prime}\right)$. Then $\theta=m g u(p(X, Y)$, $\left.p\left(X^{\prime}, Y^{\prime}\right)\right)=\left\{X^{\prime} / X, Y^{\prime} / Y\right\}$ and $\leftarrow q(X)$ is the corresponding resolvent. Now $\eta=$ $\{X / f(Y)\}$ is a c.a.s. of $P \cup\{\leftarrow q(X)\}$, but $\eta$ is not a c.a.s. of $P \cup\{\leftarrow p(X, Y)\}$.

Definition 2.4 Let A, B be conjuncts and let $\theta$ and $\sigma$ substitutions. We say that $(\mathbf{A}, \mathbf{B}, \theta, \sigma)$ is a good tuple if the following conditions are satisfied:

(i) $\operatorname{Ran}(\theta) \cap \operatorname{Var}(\mathbf{B}) \subseteq \operatorname{Var}(\mathbf{A})$ (the variables introduced by $\theta$ that occur in $\mathbf{B}$ also occur in $\mathbf{A}$ ),

(ii) $\operatorname{Ran}(\sigma) \cap(\operatorname{Var}(\mathbf{A}, \mathbf{B}) \cup \operatorname{Ran}(\theta)) \subseteq \operatorname{Var}(\mathbf{B} \theta)$

(the variables introduced by $\sigma$ that occur in $\mathbf{A}, \mathbf{B}$ or in $\operatorname{Ran}(\theta)$ also occur in $\mathbf{B} \theta$ ). 
The importance of this, admittedly esoteric, notion is revealed by the following lemma, which characterizes c.a.s.'s of a conjunction of goals in terms of c.a.s.'s of its conjuncts.

Lemma 2.5. (Good Tuple) Consider a goal $\leftarrow \mathbf{A}, \mathbf{B}$. Then $\eta$ is a c.a.s. of $P \cup\{\leftarrow \mathbf{A}, \mathbf{B}\}$ iff for some $\theta$ and $\sigma$

- $\theta$ is a c.a.s. of $P \cup\{\leftarrow \mathbf{A}\}$,

- $\sigma$ is a c.a.s. of $P \cup\{\leftarrow \mathbf{B} \theta\}$,

- $\eta=(\theta \sigma) \mid(\mathbf{A}, \mathbf{B})$,

- $(\mathbf{A}, \mathbf{B}, \theta, \sigma)$ is a good tuple.

Proof. The proof is lengthy and tedious and can be found in the technical report [4].

This lemma shows that the c.a.s.'s for a compound goal $\leftarrow \mathbf{A}$, $\mathbf{B}$ cannot be obtained by simply composing each c.a.s. $\theta$ for $\leftarrow \mathbf{A}$ with each c.a.s. $\sigma$ for $\leftarrow \mathbf{B} \theta$. The notion of a good tuple formalizes the conditions that $\theta$ and $\sigma$ have to satisfy, due to the standardization apart. Both conditions of Definition 2.4 of Good Tuple are needed: consider for example the program $P: \mathrm{p}(Z) \leftarrow$. and the goal $G=\leftarrow p(X)$, $p(Y)$. Then $\theta=\{X / Y\}$ is a c.a.s. for $\leftarrow p(X), \sigma=\varepsilon$ is a c.a.s. of $P \cup\{\leftarrow p(Y) \theta\}$ but $(\theta \sigma) \mid G=\{X / Y\}$ is not a c.a.s. of $P \cup\{G\}$. This shows that the first condition in Definition 2.4 of good tuple is needed. Now $\theta=\varepsilon$ is also a c.a.s. for $\leftarrow p(X)$, $\sigma=\{Y / X\}$ is a c.a.s. of $P \cup\{\leftarrow p(Y) \theta\}$ but $(\theta \sigma) \mid G=\{Y / X\}$ is not a c.a.s. of $P \cup\{G\}$. This shows that the second condition in Definition 2.4 of good tuple is needed.

Since we want to model the meaning of a conjunct w.r.t. a post-substitution $\eta$ in such a way that a precise match with the procedural semantics is maintained, the notion of a good tuple will be crucial also for the semantic considerations.

The next step is dictated by the simplicity considerations. We shall restrict our attention to Prolog programs in a certain form. Then, after proving soundness and completeness for these programs, we shall return to the general case.

\section{Definition 2.6. (Homogeneous Programs)}

- A Prolog clause is called homogeneous if its head is an elementary atom.

- A Prolog program is called homogeneous if all its clauses are homogeneous.

We now define truth in $\Theta$-interpretations for homogeneous programs. It relies on the notion of good tuple. Given a conjunct $\mathbf{A}$ of atoms we denote by $l(\mathbf{A})$ its length, i.e. the number of atoms in $\mathbf{A}$. If $l(\mathbf{A})=0$ we denote $\mathbf{A}$ by true.

Definition 2.7. (Truth in $\Theta$-interpretations) Let $\mathscr{I}$ be a $\Theta$-interpretation of a homogeneous Prolog program $P$.

The truth of a conjunct $\mathbf{A}$ in $\mathscr{I}$ w.r.t. a (post-)substitution $\eta$, denoted by $\mathscr{I}=\langle\mathbf{A}, \eta\rangle$, is defined by induction on $l(\mathbf{A})$, the length of $\mathbf{A}$.

- $l(\mathbf{A})=0$. Then $\mathbf{A}=$ true

$\mathscr{I} \models\langle$ true,$\eta\rangle$ iff $\eta=\varepsilon$.

- $l(\mathbf{A})=1$. Then $\mathbf{A}=A$ for an atom $A$.

$\mathscr{I} \models\langle A, \eta\rangle$ iff $\langle A, \eta\rangle \in \llbracket p \rrbracket$, where $A$ is a built-in atom with the relation symbol $p$,

$\mathscr{I} \models\langle A, \eta\rangle$ iff $\langle A, \eta\rangle \in \mathscr{I}$, where $A$ is defined in $P$. 
- $l(\mathbf{A})>1$. Then $\mathbf{A}=A, \mathbf{B}$ for an atom $A$ and a non-empty conjunct $\mathbf{B}$.

$\mathscr{I}=\langle A, \mathbf{B}, \eta\rangle$ iff there exist $\theta, \sigma$ s.t. $\eta=(\theta \sigma) \mid(A, \mathbf{B})$ and

$-\mathscr{I} \models\langle A, \theta\rangle$,

$-\mathscr{I}=\langle\mathbf{B} \theta, \sigma\rangle$

$-(A, \mathbf{B}, \theta, \sigma)$ is a good tuple.

The truth of a homogeneous clause $H \leftarrow \mathbf{B}$ of $P$ in $\mathscr{I}$, denoted by $\mathscr{I} \models H \leftarrow \mathbf{B}$, is defined as follows.

- $\mathscr{I}=\langle H \leftarrow \mathbf{B}, \eta\rangle$ iff for all $\theta$ s.t. $\operatorname{Dom}(\theta)=\operatorname{Var}(H), \operatorname{Ran}(\theta) \cap \operatorname{Var}(H \leftarrow \mathbf{B})=\varnothing$, $\operatorname{Ran}(\eta) \cap \operatorname{Var}(H \theta) \subseteq \operatorname{Var}(\mathbf{B} \theta)$ :

$\mathscr{I} \vDash\langle\mathbf{B} \theta, \eta\rangle$ implies $\mathscr{I} \models\langle H \theta, \eta \mid H \theta\rangle$,

- $\mathscr{I} \vDash H \leftarrow \mathbf{B}$ iff for all $\eta, \mathscr{I} \models\langle H \leftarrow \mathbf{B}, \eta\rangle$.

$\mathscr{I}$ is a $\Theta$-model of $P$ iff all variants of the clauses of $P$ are true in $\mathscr{I}$.

Notice that in the definition of the truth of a clause the restrictions on $\theta$ and $\sigma$ are needed in order to establish the correspondence with the operational semantics. These restrictions model at the declarative level the restrictions induced by the standardization apart. The following lemmas will be useful to reason about the truth.

Lemma 2.8. (Monotonicity) Let $\mathscr{I}, J$ be $\Theta$-interpretations, A a conjunct, and $\eta$ a substitution. If $\mathscr{I} \models\langle\mathbf{A}, \eta\rangle$ and $\mathscr{I} \subseteq J$, then $J \models\langle\mathbf{A}, \eta\rangle$.

Proof. Straightforward by induction on the length of $\mathbf{A}$.

Lemma 2.9. (Continuity) Let $\mathscr{I}_{i}(i \geqq 0)$ be $\Theta$-interpretations such that $\mathscr{I}_{0} \leqq \mathscr{I}_{1} \subseteq \ldots$. Then for every conjunct $\mathbf{A}$ and substitution $\eta$

$$
\bigcup_{i=0}^{\infty} \mathscr{I}_{i} \vDash\langle\mathbf{A}, \eta\rangle \text { iff for some } k \geqq 0 \mathscr{I}_{k} \vDash\langle\mathbf{A}, \eta\rangle \text {. }
$$

Proof. Straightforward by induction on the length of $\mathbf{A}$ and the Monotonicity Lemma 2.8 .

Note that the Continuity Lemma strengthens the Monotonicity Lemma.

\section{$2.3 \Theta$-semantics and LD-resolution}

The next step is to show that LD-resolution is correct w.r.t. the $\Theta$-semantics. The proof relies on the Good Tuple Lemma 2.5.

The following assumption is convenient.

Assumption 2.10. Whenever in the LD-resolution step the selected atom $A$ is unified with the head $H$ of the input clause where $H$ is a pure atom, then the $m g u \theta$ of $A$ and $H$ is s.t. $\operatorname{Dom}(\theta)=\operatorname{Var}(H)$.

By the previous assumption we have $A=H \theta$.

Theorem 2.11. (Soundness I) Let $P$ be a homogeneous Prolog program and $\mathbf{A}$ a conjunct. If $\eta$ is a c.a.s. for $P \cup\{\leftarrow \mathbf{A}\}$ then for any $\Theta$-model $\mathscr{I}$ of $P$ we have $\mathscr{I} \models\langle\mathbf{A}, \eta\rangle$.

Proof. Fix a $\Theta$-model $\mathscr{I}$ of $P$. Let $\xi$ be a LD-refutation of $P \cup\{\leftarrow \mathbf{A}\}$ with c.a.s. $\eta$. We prove the claim by induction on the length $l(\xi)$ of $\xi$. Three cases arise. 
Case 1. $l(\mathbf{A})=0$. Then $\mathbb{A}=$ true and $\eta=\varepsilon$, so the claim follows directly by Definition 2.7.

Case 2. $l(\mathbf{A})=1$. Then $\mathbf{A}=A$ for an atom $A$.

If $A$ is a built-in atom, then the claim follows directly by Definitions 2.1 and 2.7 .

If $A$ is defined in $P$, then consider the resolvent $\mathbf{B} \theta$ of $\leftarrow A$ in $\xi$ obtained using the input clause $H \leftarrow \mathbf{B}$ and mgu $\theta$. $H$ is a pure atom and by the standardization apart $A$ and $H \leftarrow \mathbf{B}$ have no variable in common, so by Assumption 2.10.

and

$$
\operatorname{Dom}(\theta)=\operatorname{Var}(H), \operatorname{Ran}(\theta) \cap \operatorname{Var}(H \leftarrow \mathbf{B})=\varnothing,
$$

$$
A=H \theta \text {. }
$$
Then

Let $\eta^{\prime}$ be the c.a.s. for $P \cup\{\leftarrow \mathbf{B} \theta\}$ computed by the suffix $\xi^{\prime}$ of $\xi$ starting at $\leftarrow \mathbf{B} \theta$.

$$
\eta=\left(\theta \eta^{\prime}\right) \mid A \text {. }
$$

We have $l\left(\xi^{\prime}\right)=l(\xi)-1$, so by the induction hypothesis $\mathscr{I} \vDash\left\langle\mathbf{B} \theta, \eta^{\prime}\right\rangle$. But $\mathscr{I}$ is a model of $P$, so $H \leftarrow \mathbf{B}$ is true in $\mathscr{I}$ and consequently by (1) and Definition 2.7 $\mathscr{I} \models\left\langle H \theta, \eta^{\prime} \mid H \theta\right\rangle$. Thus by (2) $\mathscr{I} \models\left\langle A, \eta^{\prime} \mid A\right\rangle$. However, $A$ and $H$ have no variable in common, so by (1) $\theta \mid A=\varepsilon$ and consequently by (3) $\eta=\left(\theta \eta^{\prime}\right)\left|A=\eta^{\prime}\right| A$. So we proved $\mathscr{I} \models\langle A, \eta\rangle$.

Case 3. $l(\mathbf{A})>1$. Then $\mathbf{A}=A, \mathbf{B}$ for an atom $A$ and a non-empty conjunct $\mathbf{B}$. By the Good Tuple Lemma 2.5 there exist $\theta$ and $\sigma$ s.t. $\eta=(\theta \sigma) \mid \mathbf{A}$ and

(i) $P \cup\{\leftarrow A\}$ has an LD-refutation $\xi_{1}$ with c.a.s. $\theta$,

(ii) $P \cup\{\leftarrow \mathbf{B} \theta\}$ has an LD-refutation $\xi_{2}$ with c.a.s. $\sigma$,

(iii) $(A, \mathbf{B}, \theta, \sigma)$ is a good tuple.

Moreover by the proof of the same Lemma it follows that we can choose $\xi_{1}, \xi_{2}$ to be subderivations of $\xi$. Then $l\left(\xi_{1}\right)<l(\xi)$ so by the induction hypothesis

$$
\mathscr{I} \models\langle A, \theta\rangle \text {. }
$$

Also $l\left(\xi_{2}\right)<l(\xi)$ so by the induction hypothesis

$$
\mathscr{I} \models\langle\mathbf{B} \theta, \sigma\rangle .
$$

Thus by (iii), (4) and (5) we get $\mathscr{I} \models\langle\mathbf{A}, \eta\rangle$ by Definition 2.7.

In order to prove the converse of Theorem 2.11 it is helpful to consider a special $\Theta$-model representing all $\Theta$-models, in the sense that a conjunction is true in it (w.r.t. a given post-substitution) iff it is true in all the $\Theta$-models.

The $\Theta$-interpretations are naturally ordered by the set inclusion. In this ordering the least $\Theta$-interpretation is $\varnothing$, the greatest one is $\Theta_{p}$. Analogously to standard Herbrand models, the $\Theta$-models are closed w.r.t. arbitrary intersections, from which we deduce the existence of the least $\Theta$-model.

Theorem 2.12. Let $P$ be a homogeneous program. Let $\mathscr{M}$ be a class of $\Theta$-models of $P$. Then $M=\cap . M I$ is a model of $P$.

Proof. Let $H \leftarrow \mathbf{B}$ be a variant of a clause of $P$ and let $\eta, \theta$ be such that $\operatorname{Dom}(\theta)=$ $\operatorname{Var}(H), \operatorname{Ran}(\theta) \cap \operatorname{Var}(H \leftarrow \mathbf{B})=\varnothing, \operatorname{Ran}(\eta) \cap \operatorname{Var}(H \theta) \subseteq \operatorname{Var}(\mathbf{B} \theta)$ and $M \models\langle\mathbf{B} \theta, \eta\rangle$. Fix $\mathscr{I} \in \mathscr{M}$. By the Monotonicity Lemma 2.8 we have $\mathscr{I}=\langle\mathbf{B} \theta, \eta\rangle$, so since $\mathscr{I}$ is a 
$\Theta$-model, $\mathscr{I} \models\langle H \theta, \eta \mid(H \theta)\rangle$. By Definition 2.7 and the fact that $\mathscr{I}$ is an arbitrary element of $\mathscr{U}$ we conclude $M \models\langle H \theta, \eta \mid H \theta\rangle$.

Corollary 2.13. (Least Model) Every homogeneous program $P$ has a least $\Theta$-model, $N_{P}$.

This $\Theta$-model is the intended representant of all $\Theta$-models of $P$ in the following sense.

Corollary 2.14. Let $\mathbf{A}$ be a conjunct and $\eta$ be a substitution. Then $N_{P} \models\langle\mathbf{A}, \eta\rangle$ iff for all $\Theta$-models $\mathscr{I}$ of $P$ we have $\mathscr{I} \models\langle\mathbf{A}, \eta\rangle$.

Proof. By the Monotonicity Lemma 2.8 .

In the theory of Logic Programming the least Herbrand model can be generated as the least fixpoint of the immediate consequence operator $T_{P}$ on the Herbrand interpretations. This characterization is useful to establish the completeness of SLD-resolution with respect to the least Herbrand model. We now provide an analogous characterization of the least $\Theta$-model $N_{P}$ in order to show the completeness of the LD-resolution with respect to $N_{P}$.

First, we introduce the appropriate operator $T_{P}$.

Definition 2.15. Let $P$ be a homogeneous program. The immediate consequence operator $T_{P}$ on the $\Theta$-interpretations is defined as follows

$$
\begin{aligned}
T_{P}(\mathscr{I})= & \{\langle H \theta, \eta \mid(H \theta)\rangle \mid \text { for some } \mathbf{B} \\
& H \leftarrow \mathbf{B} \text { is a variant of a clause from } P, \\
& \operatorname{Dom}(\theta)=\operatorname{Var}(H), \operatorname{Ran}(\theta) \cap \operatorname{Var}(H \leftarrow \mathbf{B})=\varnothing, \\
& \operatorname{Ran}(\eta) \cap \operatorname{Var}(H \theta) \subseteq \operatorname{Var}(\mathbf{B} \theta), \mathscr{I} \models\langle\mathbf{B} \theta, \eta\rangle\} .
\end{aligned}
$$

Next, we characterize the $\Theta$-models of $P$ as the pre-fixpoints of $T_{P}$. The following proposition shows this characterization for programs consisting of one clause only.

Proposition 2.16. Given a clause $C$ and $a \Theta$-interpretation $\mathscr{I}$, we have that $\mathscr{I}$ is a model of $\{C\}$ iff $T_{\{C\}}(\mathscr{I}) \subseteq \mathscr{I}$.

Proof. For every $H, \theta$ and $\eta$ we have $\langle H \theta, \eta \mid H \theta\rangle \in T_{\{C,}(\mathscr{I})$ iff (by Definition 2.15) $H \leftarrow \mathbf{B}$ is a variant of $C$ such that $\mathscr{I} \models\langle\mathbf{B} \theta, \eta\rangle, \operatorname{Dom}(\theta)=\operatorname{Var}(H), R$ an $(\theta) \cap$ $\operatorname{Var}(H \leftarrow \mathbf{B})=\varnothing$ and $\operatorname{Ran}(\eta) \cap \operatorname{Var}(H \theta) \subseteq \operatorname{Var}(\mathbf{B} \theta)$. Since $\mathscr{I}$ is a model of $\{C\}$ then this holds iff $\mathscr{I} \models\langle H \theta, \eta \mid(H \theta)\rangle$, i.e. $\langle H \theta, \eta \mid(H \theta)\rangle \in \mathscr{I}$.

To generalize Proposition 2.16 to non-singleton programs we use the following obvious lemma which states the additivity of the operator $T_{P}$.

Lemma 2.17. Let $P, P^{\prime}$ be homogeneous programs. Then for every $\Theta$-interpretation $\mathscr{I}$ we have $T_{P \cup P^{\prime}}(\mathscr{I})=T_{P}(\mathscr{I}) \cup T_{P^{\prime}}(\mathscr{I})$.

Corollary 2.18. (Model Characterization) $\mathscr{I}$ is a $\Theta$-model of $P$ iff $T_{P}(\mathscr{I}) \subseteq \mathscr{I}$.

Now, we characterize $N_{P}$ as the least fixpoint of $T_{P}$. We need the following observation.

Proposition 2.19.(Monotonicity) $T_{P}$ is monotonic, that is $I \subseteq J$ implies $T_{P}(I) \subseteq T_{P}(J)$.

Proof. By the Monotonicity Lemma 2.8 . 
Proposition 2.20. (Least Fixpoint) $T_{P}$ has a least fixpoint lfp $\left(T_{P}\right)$ which is also its least pre-fixpoint.

Proof. By the Monotonicity Proposition 2.19 and Knaster-Tarski Theorem.

We can now derive the desired result.

Corollary 2.21. If $\left(T_{P}\right)=N_{P}$.

Proof. By the Least Fixpoint Proposition 2.20, Least Model Corollary 2.13 and Model Characterization Corollary 2.18.

Finally, we provide a more precise characterization of the $\Theta$-model $N_{P}$ that will be used in the proof of the completeness of the LD-resolution. We need the following strengthening of the Monotonicity Proposition 2.19.

Proposition 2.22. (Continuity) $T_{P}$ is continuous, that is for every sequence $\mathscr{I}_{i}(i \geqq 0)$ of $\Theta$-interpretations such that $\mathscr{I}_{0} \subseteq \mathscr{I}_{1} \subseteq \ldots$ we have

$$
T_{P}\left(\bigcup_{i=0}^{\infty} \mathscr{I}_{i}\right)=\bigcup_{i=0}^{\infty} T_{P}\left(\mathscr{I}_{i}\right)
$$

Proof. By the Continuity Lemma 2.9 .

We define now a sequence of $\Theta$-interpretations by

$$
\begin{aligned}
T_{P} \uparrow 0 & =\varnothing, \\
T_{P} \uparrow(n+1) & =T_{P}\left(T_{P} \uparrow n\right), \\
T_{P} \uparrow w & =\bigcup_{i=0}^{\infty} T_{P} \uparrow i .
\end{aligned}
$$

Proposition 2.23. (Characterization) $N_{P}=T_{P} \uparrow \omega$.

Proof. By the Continuity Proposition 2.22 and the Knaster-Tarski Theorem $l f p\left(T_{P}\right)=$ $T_{P} \uparrow \omega$, so the claim follows by Corollary 2.21 .

We can now prove the completeness of LD-resolution with respect to the $\Theta$-semantics for homogeneous programs.

Theorem 2.24. (Completeness I) Consider a homogeneous program $P$ and a conjunct A. Suppose that for all $\Theta$-models $\mathscr{I}$ of $P$ we have $\mathscr{I} \vDash\langle\mathbf{A}, \eta\rangle$. Then there exists an LD-refutation of $P \cup\{\leftarrow \mathbf{A}\}$ with c.a.s. $\eta$.

Proof. In particular we have $N_{P} \models\langle\mathbf{A}, \eta\rangle$. By the Characterization Proposition $2.23 T_{P} \uparrow \omega \models\langle\mathbf{A}, \eta\rangle$. By the monotonicity of $T_{P}$ we have $T_{P} \uparrow 0 \subseteq T_{P} \uparrow 1 \subseteq \ldots$, so by the Continuity Lemma $2.9 T_{\boldsymbol{P}} \uparrow k \models\langle\mathbf{A}, \eta\rangle$ for some $\left.k\right\rangle 0$.

We now prove the claim by induction w.r.t. the lexicographic ordering $<$ defined on pairs $\langle k, l(\mathbf{A})\rangle$ of natural numbers. In this ordering

$$
\left\langle n_{1}, n_{2}\right\rangle<\left\langle m_{1}, m_{2}\right\rangle \text { iff }\left(n_{1}<m_{1}\right) \text { or }\left(n_{1}=m_{1} \wedge n_{2}<m_{2}\right) \text {. }
$$

The case when $\mathbf{A}$ is empty, i.e. $l(\mathbf{A})=0$ (which covers the base case of the induction) is immediate by Definition 2.7.

Suppose now $\mathbf{A}=A, \mathbf{B}$. There exist substitutions $\theta, \sigma$ such that

$$
\begin{aligned}
& T_{P} \uparrow k \models\langle A, \theta\rangle, \\
& T_{P} \uparrow k \models\langle\mathbf{B} \theta, \sigma\rangle,
\end{aligned}
$$

$(A, \mathbf{B}, \theta, \sigma)$ is a good tuple and $\eta=(\theta \sigma) \mid(A, \mathbf{B})$. 
We first prove that $P \cup\{\leftarrow A\}$ has an LD-refutation with c.a.s. $\theta$. When $A$ is a built-in atom this conclusion follows immediately from Definitions 2.1 and 2.7.

When $A$ is defined in $P$ we have $k>0$. By Definition 2.15 there exists a variant $H \leftarrow \mathbb{B}^{\prime}$ of a clause from $P$, a substitution $\psi$ s.t. $\operatorname{Dom}(\psi)=\operatorname{Var}(H), \operatorname{Ran}(\psi) \cap$ $\operatorname{Var}\left(H \leftarrow \mathbf{B}^{\prime}\right)=\varnothing, A=H \psi$ and a substitution $\phi$ such that

$$
\operatorname{Ran}(\phi) \cap \operatorname{Var}(H \psi) \cong \operatorname{Var}(\mathbf{B} \psi),
$$

$T_{P} \uparrow(k-1) \models\left\langle\mathbf{B}^{\prime} \psi, \phi\right)$ and $\theta=\phi \mid A$.

Since $\left\langle k-1, l\left(\mathbf{B}^{\prime} \psi\right)\right\rangle\langle\langle k, l(\mathbf{A})\rangle$, by the induction hypothesis there exists an LD-refutation of $P \cup\left\{\leftarrow \mathbf{B}^{\prime} \psi\right\}$ with c.a.s. $\phi$. Now notice that $\operatorname{Dom}(\psi)=\operatorname{Var}(H)$, $\leftarrow \mathbf{B}^{\prime} \psi$ is a resolvent of $\leftarrow A$ using the $m g u \psi$ and (6) holds. Then by the c.a.s. Lemma $2.3 \theta$ is a c.a.s. of $P \cup\{\leftarrow A\}$.

Since $\langle k, l(\mathbf{B} \theta)\rangle\langle\langle k, l(\mathbf{A})\rangle$, by the induction hypothesis also there exists an LD-refutation of $P \cup\{\leftarrow \mathbf{B} \theta\}$ with c.a.s. $\sigma$. Since $(A, \mathbf{B}, \theta, \sigma)$ is a good tuple and $\eta=(\theta \sigma) \mid(A, \mathbf{B})$, we can apply the Good Tuple Lemma 2.5. We conclude that there exists an LD refutation of $P \cup\{\leftarrow \mathbf{A}\}$ with c.a.s. $\eta$.

Corollary 2.25. Let $P$ be a homogeneous Prolog program. Then

$$
\begin{aligned}
& N_{P}=\{\langle A, \eta\rangle \mid A \text { is defined in } P \text { and } \\
& \quad \text { there exists an LD-refutation of } P \cup\{\leftarrow A\} \text { with c.a.s. } \eta\} .
\end{aligned}
$$

Proof. By Definition 2.7 and Theorems 2.11 and 2.24.

This corollary shows that the $\Theta$-model $N_{P}$ captures precisely the computational meaning of the homogeneous program $P$.

\subsection{Extension to Arbitrary Programs}

Now, every program can be easily transformed into a homogeneous program.

Definition 2.26. (Homogeneous Form) Let $P$ be a Prolog program. Let $x_{1}, x_{2}, \ldots$ be distinct variables not occurring in $P$. Transform each clause

$$
p\left(t_{1}, \ldots, t_{k}\right) \leftarrow \mathbf{B}
$$

of $P$ into the clause

$$
p\left(x_{1}, \ldots, x_{k}\right) \leftarrow x_{1}=t_{1}, \ldots, x_{k}=t_{k}, \mathbf{B} .
$$

Here $=$ is the built-in discussed in Sect. 2 and interpreted as "is unifiable with". We denote the resulting program by $\operatorname{Hom}(P)$ and call it a homogeneous form of $P$.

We now show that a Prolog program $P$ and its homogeneous form $\operatorname{Hom}(P)$ have the same computational behaviour.

Theorem 2.27. (Equivalence I) Let $P$ be a Prolog program, $G$ a goal. Then $P \cup\{G\}$ has a refutation with c.a.s. $\eta$ if and only if $\operatorname{Hom}(P) \cup\{G\}$ has a refutation with c.a.s. $\eta$.

Proof. See [4].

Theorem 2.27 allows to reason about the meaning of Prolog programs by transforming them first to a homogeneous form. Alternatively, we can extend the definition of the truth to arbitrary programs by simply defining a clause to be true 
iff its homogeneous version is true. By "processing" then the meaning of the introduced calls to the built-in = we obtain the following direct definition of truth of a clause.

Definition 2.28. $\mathscr{I} \models\langle H \leftarrow \mathbf{B}, \eta\rangle$ iff for any atom $A$ and a variant $H^{\prime} \leftarrow \mathbf{B}^{\prime}$ of $H \leftarrow \mathbf{B}$ disjoint from $A$ the following implication holds: $\theta=m g u\left(A, H^{\prime}\right), \mathscr{I} \vDash\left\langle\mathbf{B}^{\prime} \theta, \eta\right\rangle$ and $\operatorname{Ran}(\eta) \cap\left(\operatorname{Var}(A) \cup \operatorname{Var}\left(H^{\prime} \leftarrow \mathbb{B}^{\prime}\right)\right) \subseteq \operatorname{Var}\left(\mathbf{B}^{\prime} \theta\right)$ implies $\mathscr{I} \models\langle A, \theta \eta \mid A\rangle$.

We now establish the semantics equivalence of a program and its homogeneous form.

Theorem 2.29. (Equivalence II) $\mathscr{I}$ is a model of a Prolog program $P$ iff it is a model of $\operatorname{Hom}(P)$.

Proof. See [4].

From the two previous results on operational and semantic equivalence of $P$ and $\operatorname{Hom}(P)$ the soundness and completeness of the LD-resolution for Prolog programs directly follows.

Theorem 2.30. (Soundness II) Let $P$ be a Prolog program and $\mathbf{A}$ a conjunct. If $\eta$ is a c.a.s. for $P \cup\{\leftarrow \mathbf{A}\}$ then for any $\Theta$-model $\mathscr{I}$ of $P$ we have $\mathscr{I} \models\langle\mathbf{A}, \eta\rangle$.

Proof. By the Equivalence I Theorem 2.27 and the Equivalence II Theorem 2.29.

Theorem 2.31 (Completeness II) Consider a Prolog program $P$ and a conjunct $\mathbf{A}$. Suppose that for all $\Theta$-models $\mathscr{I}$ of $P$ we have $\mathscr{I} \models\langle\mathbf{A}, \eta\rangle$. Then there exists an $L D$-refutation of $P \cup\{\leftarrow \mathbf{A}\}$ with c.a.s. $\eta$.

Proof. By the Equivalence II Theorem 2.29 and the Equivalence I Theorem 2.27.

\subsection{Relation between the $\Theta$-Semantics and the S-Semantics}

In this section we show that the $\Theta$-semantics is the natural extension to Prolog programs of the $S$-Semantics defined in Falaschi et al. [12] for logic programs, in the sense that if $P$ is a pure Prolog program (i.e. it does not contain built-in atoms) then the least $\Theta$-model $N_{P}$ coincides with the least $S$-model $S_{P}$. To this purpose, it will be helpful to consider the following operational characterization of $S_{P}$ (cf. Falaschi et al. [12]).

$$
S_{P}=\{p(\mathbf{x}) \eta \mid \mathbf{x} \in \operatorname{Var} \text { and } \leftarrow p(\mathbf{x}) \text { has an LD-refutation with c.a.s. } \eta\}
$$

or, equivalently,

$$
S_{P}=\{\langle p(\mathbf{x}), \eta\rangle \mid \mathbf{x} \in \text { Var and } \leftarrow p(\mathbf{x}) \text { has an LD-refutation with c.a.s. } \eta\}
$$

We define now some properties on $\Theta$-interpretations which will be shown to hold for $N_{P}$ when $P$ is pure, and which will be useful for proving the correspondence stated above.

Definition 2.32. Let $\mathscr{I}$ be a $\Theta$-interpretation. $\mathscr{I}$ is called

- upward-closed iff $\forall\langle A, \eta\rangle \in \mathscr{I}, \forall \theta$ such that $\exists \sigma=m g u(A \theta, A \eta)$, we have $\left\langle A \theta, \sigma^{\prime}\right\rangle \in \mathscr{I}$, where $\sigma^{\prime}$ is the restriction of $\sigma$ to $A \theta$.

- downward-closed iff $\forall\langle A \theta, \sigma\rangle \in \mathscr{I} . \exists \eta . \exists \sigma^{\prime}=m g u(A \theta, A \eta)$. $\langle A, \eta\rangle \in \mathscr{I}$ and $\sigma$ is the restriction of $\sigma^{\prime}$ to $A \theta$. 
Proposition 2.33 Let $P$ be a pure Prolog program. Then $N_{P}$ is upward-closed and downward-closed.

Proof. By using the characterization of $N_{P}$ expressed by Corollary 2.25 it is sufficient to prove the operational counterparts of upward and downward closedness, which when extended to arbitrary conjunctions, are expressed by the following lemma.

\section{Lemma 2.34}

1. If the goal $\leftarrow \mathbf{Q}$ has a $L D$-refutation with c.a.s. $\eta$, then, for each $\theta$ such that $\exists \sigma=m g u(\mathbf{Q} \theta, \mathbf{Q} \eta)$, the goal $\leftarrow \mathbf{Q} \theta$ has an LD-refutation with a computed answer substitution $\sigma^{\prime}$ which is the restriction of $\sigma$ to $\mathbf{Q} \theta$.

2. If the goal $\leftarrow \mathbf{Q} \theta$ has a LD-refutation with c.a.s. $\sigma$ then there exists $\eta$ and $\sigma^{\prime}=\operatorname{mgu}(\mathbf{Q} \theta, \mathbf{Q} \eta)$ such that $\leftarrow \mathbf{Q}$ has a LD-refutation with c.a.s. $\eta$ and $\sigma$ is the restriction of $\sigma^{\prime}$ to $\mathbf{Q} \eta$.

Proof. See [4]

Note the analogy between Lemma 2.34(2) and the Lifting Lemma. Actually, Lemma 2.34(2) (which can obviously be generalized to arbitrary selection rules) is stronger than Lifting Lemma, because not only it ensures the existence of $\eta$, but it also gives more precise information about the relation between $\theta, \sigma$ and $\eta$ (from the Lifting Lemma we would only know that $\mathbf{Q} \eta \leqq \mathbf{Q} \theta \sigma)$.

If $P$ contains built-in relations, then $N_{P}$ could be non upward-closed or non downward-closed.

Example 2.35 Consider the program $P$ :

$$
\begin{aligned}
& p(X) \leftarrow \operatorname{var}(X), q(X) . \\
& q(a) \leftarrow .
\end{aligned}
$$

The goal $\leftarrow p(x)$ has an LD-refutation with c.a.s. $\eta=\{x / a\}$, but the goal $\leftarrow p(x) \eta$ has no refutations. Thus, $N_{P}$ is not upward-closed.

Consider the program $P$ :

$\mathrm{p}(\mathrm{X}) \leftarrow$ nonvar $(\mathrm{X})$.

The goal $\leftarrow p(a)$ has an LD-refutation, but the goal $\leftarrow p(x)$ has no refutations. Thus, $N_{P}$ is not downward-closed.

We show now that if $P$ is a pure Prolog program, then $N_{P}$ is isomorphic to the least $S$-model $S_{P}$, in the sense that there exist a mapping $\alpha$ from $S$-interpretations to $\Theta$-interpretations, and a mapping $\beta$ from $\Theta$-interpretations to $S$-interpretations such that for every program $P N_{P}=\alpha\left(S_{P}\right)$ and $S_{P}=\beta\left(N_{P}\right)$.

Note that $\alpha$ and $\beta$ are abstraction operators, i.e. they do not depend upon $P$ : if $S_{P_{1}}=S_{P_{2}}$ then $\beta\left(S_{P_{1}}\right)=\beta\left(S_{P_{2}}\right)$ and if $N_{P_{1}}=N_{P_{2}}$ then $\alpha\left(N_{P_{1}}\right)=\alpha\left(N_{P_{2}}\right)$.

Definition 2.36. The mappings $U p$ from $S$-interpretations to $\Theta$-interpretations, and Kernel from $\Theta$-interpretations to $S$-interpretations are defined as follows.

$U p(\mathscr{I})=\left\{\langle A \theta, \sigma\rangle \mid \exists \eta \cdot\langle A, \eta\rangle \in \mathscr{I}\right.$ and $\exists \sigma^{\prime}=m g u(A \theta, A \eta) . \sigma$ is the restriction of $\sigma^{\prime}$ to $\left.A \theta\right\}, \operatorname{Kernel}(\mathscr{I})=\{\langle p(\mathbf{x}), \eta\rangle \mid \mathbf{x} \in \operatorname{Var}$ and $\langle p(\mathbf{x}), \eta\rangle \in \mathscr{I}\}$

Note that the definition of $U p$ and Kernel does not depend upon $P$. We prove that $U p$ and Kernel are the intended $\alpha$ and $\beta$ satisfying the property described above. 
Proposition 2.37. If $P$ is a pure Prolog program, then

1. $N_{P}=U p\left(S_{P}\right)$, and

2. $S_{P}=\operatorname{Kernel}\left(N_{P}\right)$.

Proof. The equality $S_{P}=\operatorname{Kernel}\left(N_{P}\right)$ follows immediately by the definition of Kernel and by (7). Therefore we have only to prove that $N_{P}=U p\left(\operatorname{Kernel}\left(N_{P}\right)\right)$.

$\cong)$ Let $\langle A, \sigma\rangle \in N_{P}$. Assume $A=p(\mathbf{x}) \theta$. Then by Proposition 2.33(2) there exist $\eta$ and $\sigma^{\prime}=m g u(A, p(\mathbf{x}) \eta)$ such that $\langle p(\mathbf{x}), \eta\rangle \in N_{P}$ (and therefore $\langle p(\mathbf{x}), \eta\rangle \in$ $\left.\operatorname{Kernel}\left(N_{P}\right)\right)$, and $\sigma$ is the restriction of $\sigma^{\prime}$ to $A$. The rest follows by Proposition 2.33(1).

$\supseteqq)$ Let $\langle A, \sigma\rangle \in U p\left(\operatorname{Kernel}\left(N_{P}\right)\right)$. Then there exists $\langle p(\mathbf{x}), \eta\rangle \in \operatorname{Kernel}\left(N_{P}\right) \subseteq N_{P}$ such that, for some $\theta, A=p(\mathbf{x}) \theta$ and $\exists \sigma^{\prime}=m g u(A, p(\mathbf{x}) \eta)$ such that $\sigma$ is the restriction of $\sigma^{\prime}$ to $A$. By Proposition 2.33(1), we conclude $\langle A, \sigma\rangle \in N_{p}$.

\subsection{Full Abstraction of the $\Theta$-Semantics}

In the previous sections we have seen that $N_{P}$ coincides with the set of computational pairs $\langle A, \eta\rangle$ s.t. there exists an LD-refutation of $P \cup\{\leftarrow A\}$ with c.a.s. $\eta$ and that the $\Theta$-semantics is and-compositional, in the sense that the truth value of a conjunction of atoms (possibly sharing variables) can be derived by the truth value of the atoms.

We argue that a declarative semantics should provide such a compositional interpretation of conjuncts. We focus on conjuncts of the form

$$
p_{1}\left(\mathbf{x}_{1}\right), \ldots, p_{n}\left(\mathbf{x}_{n}\right)
$$

where the $p_{i}\left(\mathbf{x}_{i}\right)$ 's are either elementary atoms or atoms of the form $x=t$, and $\mathbf{x}_{1}, \ldots, \mathbf{x}_{n}$ are possibly not disjoint. Every conjunct can be equivalently transformed into a conjunct of this form.

One might wonder whether it is possible to develop a declarative semantics for Prolog based on a simpler (i.e. more abstract) domain than the $\Theta$-domain, possibly encoding less information concerning the computational behavior of goals. One might for instance be interested in observing only the non-ground success set of a program $P$, defined as:

$$
N G S S_{P}=\{A \eta \mid \leftarrow A \text { has an LD-refutation with c.a.s. } \eta\}
$$

(which corresponds to the least $\mathrm{C}$-model when $P$ is a pure program (cf. Falaschi et al. [12])). This notion can be considered the most abstract interesting one, since, as we already have seen in the introduction, the ground success set is not suitable for programs containing built-in relations. So the question is:

is it possible to give a declarative, hence and-compositional, characterization of $N G S S_{P}$ ?

If we want to have a declarative model which coincides with $N G S S_{P}$, then the answer is no. In fact, it is easy to show that $N_{G S S_{P}}$ is not and-compositional (in the sense that the $N G S S_{P}$ information about a goal in $P$ cannot be derived from the $N G S S_{P}$ information about its atomic subgoals). An example of this fact will be given below.

We have therefore to be content with a declarative semantics from which it is possible to derive $N G S S_{P}$, but which contains more information than $N G S S_{P}$ 
necessary to achieve and-compositionality. The main result of this section is that the information encoded in $N_{P}$ is the least one which is necessary to model $N G S S_{P}$ and to provide an and-compositional notion of truth. In other words, $N_{P}$ is the fully abstract semantics with respect to and-compositionality and $N G S S_{P}$, which means that $N_{P}$ is the simplest declarative semantics for Prolog programs with first-order built-in's.

We first introduce the notions of semantical mappings associated to $N_{P}$ and $N G S S_{P}$ (which we will still denote by $N_{P}$ and $N G S S_{P}$ ).

Definition 2.38. Let $\mathbf{x}_{1}, \ldots, \mathbf{x}_{n}$ be sequences of variables, possibly not disjoint. Let $p_{1}\left(\mathbf{x}_{1}\right), \ldots, p_{n}\left(\mathbf{x}_{n}\right)$ be either elementary atoms, or atoms of the form $\mathbf{x}=\mathbf{t}$.

- The mapping $N_{P}$ from conjunctions of elementary atoms to pairs of substitutions is defined as follows:

$$
\begin{aligned}
N_{P} \llbracket p_{1}\left(\mathbf{x}_{1}\right), \ldots, p_{n}\left(\mathbf{x}_{n}\right) \rrbracket=\{\langle\theta, \eta\rangle & \operatorname{Dom}(\theta) \leqq\left\{\mathbf{x}_{1}\right\} \cup \cdots \cup\left\{\mathbf{x}_{n}\right\} \text { and } \\
& \leftarrow\left(p_{1}\left(\mathbf{x}_{1}\right), \ldots, p_{n}\left(\mathbf{x}_{n}\right)\right) \theta \\
& \text { has an LD-refutation with c.a.s. } \eta\}
\end{aligned}
$$

- The mapping $N G S S_{P}$ from conjunctions of elementary atoms to substitutions is defined as follows:

$$
\begin{aligned}
N G S S_{P} \llbracket p_{1}\left(\mathbf{x}_{1}\right), \ldots, p_{n}\left(\mathbf{x}_{n}\right) \rrbracket=\{\theta \eta \mid & \operatorname{Dom}(\theta) \subseteq\left\{\mathbf{x}_{1}\right\} \cup \cdots \cup\left\{\mathbf{x}_{n}\right\} \text { and } \\
& \leftarrow\left(p_{1}\left(\mathbf{x}_{1}\right), \ldots, p_{n}\left(\mathbf{x}_{n}\right)\right) \theta \\
& \text { has an LD-refutation with c.a.s. } \eta\}
\end{aligned}
$$

The correspondence with the standard notions of $N_{P}, N G S S_{P}$ is immediate, since

and

$$
N_{P} \llbracket p(\mathbf{x}) \rrbracket=\left\{\langle\theta, \eta\rangle \mid\langle p(\mathbf{x}) \theta, \eta\rangle \in N_{P}\right\}
$$

$$
N G S S_{P} \llbracket p(\mathbf{x}) \rrbracket=\left\{\sigma \mid p(\mathbf{x}) \sigma \in N G S S_{P}\right\} .
$$

The semantics $N G S S_{P}$ is more abstract than $N_{P}$, i.e. the information encoded in $N G S S_{P}$ can be retrieved from the one in $N_{P}$ (correctness of $N_{P}$ w.r.t. $N G S S_{P}$ ). This is shown by the following fact.

Fact 1. $N G S S_{P} \llbracket \mathbf{Q} \rrbracket=\left\{\theta \eta \mid\langle\theta, \eta\rangle \in N_{P} \llbracket \mathbf{Q} \rrbracket\right\}$.

On the other hand, it is not possible to retrieve the information encoded in $N_{P}$ from the one encoded in $N G S S_{P}$, i.e. $N_{P}$ and $N G S S_{P}$ are not equivalent. This is because the mapping $N_{P}$ is and-compositional and $N G S S_{P}$ is not. In fact $N_{P} \llbracket \mathbf{Q}, \mathbf{R} \rrbracket$ can be derived from $N_{P} \llbracket \mathbf{Q} \rrbracket$ and $N_{P} \llbracket \mathbf{R} \rrbracket$ :

$$
\begin{aligned}
& \langle\theta, \eta\rangle \in N_{P} \llbracket \mathbf{Q}, \mathbf{R} \rrbracket \quad \text { iff } \exists \sigma \cdot\langle\theta, \sigma\rangle \in N_{P} \llbracket \mathbf{Q} \rrbracket \text {, and } \\
& \langle\theta \sigma, \eta\rangle \in N_{P} \llbracket \mathbf{R} \rrbracket \text {, and } \\
& (\mathbf{Q} \theta, \mathbf{R} \theta, \sigma, \eta) \text { is a good tuple. }
\end{aligned}
$$

On the contrary, $N G S S_{P}$ is not and-compositional, as it is shown in the following example.

Example 2.39. Consider the program $P$ :

$$
\begin{aligned}
& p(X) \leftarrow X=a . \\
& q(X) \leftarrow \operatorname{var}(X), X=a .
\end{aligned}
$$


We have $N G S S_{P} \llbracket p(x) \rrbracket=N G S S_{P} \llbracket q(x) \rrbracket=\{\{x / a\}\}$, but $N G S S_{P} \llbracket p(x), p(x) \rrbracket=$ $\{\{x / a\}\}$ whereas $N G S S_{P} \llbracket q(x), q(x) \rrbracket=\varnothing$. Note that the key point of this counterexample is the presence of shared variables.

The next theorem shows, however, that $N_{P}$ is the most abstract and-compositional semantics which is correct w.r.t. $N G S S_{P}$. We first need the following lemma.

Lemma 2.40. If $\langle\theta, \eta\rangle \in N_{P} \llbracket \mathbf{Q} \rrbracket \backslash N_{P} \llbracket \mathbf{R} \rrbracket$ then there exists $\left\langle\theta^{\prime}, \eta^{\prime}\right\rangle \in N_{P} \llbracket \mathbf{Q} \rrbracket \backslash N_{\boldsymbol{P}} \llbracket \mathbf{R} \rrbracket$ s.t. $\theta^{\prime}$ is idempotent.

Proof.

From $\langle\theta, \eta\rangle \in N_{P} \llbracket \mathbf{Q} \rrbracket$ it follows that there exists an $L D$-refutation $\xi$ of $P \cup\{\leftarrow \mathbf{Q} \theta\}$ with c.a.s. $\eta$. Let $\operatorname{Dom}(\theta) \cap \operatorname{Ran}(\theta)=\left\{x_{1}, \ldots, x_{n}\right\}$ and let $y_{1}, \ldots, y_{n}$ distinct variables that do not occur in $\xi$. Let $\rho=\left\{x_{1} / y_{1}, \ldots, x_{n} / y_{n}\right\}, \rho^{-1}=\left\{y_{1} / x_{1}, \ldots, y_{n} / x_{n}\right\}$. Let $\theta^{\prime}=(\theta \rho) \mid \mathbf{Q}$ and $\eta^{\prime}=\left(\rho^{-1} \eta\right) \mid\left(\mathbf{Q} \theta^{\prime}\right)$. Then $\xi^{\prime}=\xi \rho$, is an LD-refutation of $P \cup\left\{\leftarrow \mathbf{Q} \theta^{\prime}\right\}$ with c.a.s. $\eta^{\prime}$. Hence $\left\langle\theta^{\prime}, \eta^{\prime}\right\rangle \in N_{P} \llbracket \mathbf{Q} \rrbracket \backslash N_{P} \llbracket \mathbf{R} \rrbracket$ and $\theta^{\prime}$ is idempotent.

Theorem 2.41. (Full Abstraction) If $N_{P} \llbracket \mathbf{Q} \rrbracket \neq N_{P} \llbracket \mathbf{R} \rrbracket$ then there exists a conjunction A such that $N G S S_{P} \llbracket \mathbf{A}, \mathbf{Q} \rrbracket \neq N G S S_{P} \llbracket \mathbf{A}, \mathbf{R} \rrbracket$.

Proof.

Assume, without loss of generality, that there exist $\langle\theta, \eta\rangle \in N_{P} \llbracket \mathbf{Q} \rrbracket \backslash N_{P} \llbracket \mathbf{R} \rrbracket$. By Lemma 2.40 we can assume $\theta$ idemp itent. Let $\theta=\left\{x_{1} / t_{1}, \ldots, x_{m} / t_{m}\right\}, \operatorname{Var}(\mathbf{Q} \theta)=$ $\left\{y_{1}, \ldots, y_{n}\right\}$.

Define now:

$$
\begin{aligned}
& \mathbf{A}_{1}=x_{1}=t_{1}, \ldots, x_{m}=t_{m}, \\
& \mathbf{A}_{2}=\operatorname{var}\left(y_{1}\right), \ldots, \operatorname{var}\left(y_{n}\right), \\
& \mathbf{A}_{3}=\left(y_{k_{1}} \backslash==y_{l_{1}}\right), \ldots,\left(y_{k_{r}} \backslash==y_{l_{r}}\right),
\end{aligned}
$$

where $\left\{\left\{k_{1}, l_{1}\right\}, \ldots,\left\{k_{r}, l_{r}\right\}\right\}$ are all possible combinations of two indexes in the set $\{1, \ldots, n\}(r$ is the cardinality of such combinations: $r=(n-1) n / 2)$. Finally, define $\mathbf{A}=\mathbf{A}_{1}, \mathbf{A}_{2}, \mathbf{A}_{3}$. By the definition of $\mathbf{A}$ we derive immediately that $\theta \eta \in N G S S_{P} \llbracket \mathbf{A}, \mathbf{Q} \rrbracket$. We show now that $\theta \eta \notin N G S S_{P} \llbracket \mathbf{A}, \mathbf{R} \rrbracket$. Assume, by contradiction, that there exists $\sigma \in N G S S_{P} \llbracket \mathbf{A}, \mathbf{R} \rrbracket$ such that

$$
\sigma=\theta \eta
$$

Then, there exist $\phi, \psi$ such that $\leftarrow(\mathbf{A}, \mathbf{R}) \phi$ has an LD-refutation with c.a.s. $\psi$ and

$$
\phi \psi=\sigma .
$$

We show that in this case $\leftarrow \mathbf{R} \theta$ has an LD-refutation with c.a.s. $\eta$, i.e. $\langle\theta, \eta\rangle \in N_{P}(\mathbf{R})$, against the hypothesis. Consider an LD-refutation for $\leftarrow(\mathbf{A}, \mathbf{R}) \phi$ with a c.a.s. $\psi$ which satisfies (9). Then there exists $\gamma, \tau$ such that

$$
\begin{aligned}
& \leftarrow \mathbf{A}_{1} \phi \text { has an LD-refutation with c.a.s. } \gamma, \\
& \leftarrow \mathbf{A}_{2} \phi \gamma \text { has an LD-refutation (with c.a.s. } \varepsilon \text { ), } \\
& \leftarrow \mathbf{A}_{3} \phi \gamma \text { has an LD-refutation (with c.a.s. } \varepsilon \text { ), } \\
& \leftarrow \mathbf{R} \phi \gamma \text { has an LD-refutation with c.a.s. } \tau,
\end{aligned}
$$

and

$$
\gamma \tau=\psi
$$


Since $\theta$ is a mgu for $\left\{x_{1}=t_{1}, \ldots, x_{n}=t_{n}\right\}$, by (10) we have $\mathbf{A}_{1} \theta \leqq \mathbf{A}_{1} \phi \gamma$. Moreover, by (11), (12) and the definition of $\llbracket$ var $\rrbracket$ and $\llbracket \backslash==\rrbracket$ we also have $\mathbf{A}_{1} \phi \gamma \leqq \mathbb{A}_{1} \theta$, and therefore, since the domains of $\phi \gamma$ and $\theta$ are restricted to $\mathbf{A}_{1}$, we can derive (up to renaming)

$$
\phi \gamma=\theta
$$

By (13), we have that

$\leftarrow \mathbf{R} \theta$ has an LD-refutation with c.a.s. $\tau$

furthermore, by (15), (14), (9), and (8),

$$
\mathbf{R} \theta \tau=\mathbf{R} \phi \gamma \tau=\mathbf{R} \phi \psi=\mathbf{R} \sigma=\mathbf{R} \theta \eta
$$

i.e. (since both the domains of $\tau$ and $\eta$ are restricted to $\mathbf{Q} \theta$ ), $\tau=\eta$.

\section{Termination of Prolog Programs}

In this section we show that the $\Theta$-semantics is helpful when studying termination of Prolog programs. The presence of built-in's allows us to better control the execution of the programs and consequently it is not surprising that most "natural" programs with built-in's terminate for all goals. This motivates the following definition.

Definition 3.1. We say that a Prolog program $P$ strongly terminates if for all goals $G$ all LD-derivations of $P \cup\{G\}$ are finite.

Traditionally, the main concept used to prove termination of Prolog programs is that of a level mapping. Level mapping was originally defined to be a function from ground atoms to natural numbers (see Bezem [6], Cavedon [9], Apt and Pedreschi [5]).

In our case it is more natural to consider level mappings defined on non-ground atoms. Such level mappings were already considered in Bossi, Cocco and Fabris [8] and subsequently in Plümer [16] but they were applied only to prove termination of pure Prolog programs. In our case it is convenient to allow a level mapping yielding values in a well-founded ordering.

Definition 3.2. A level mapping || is a function from atoms to a well-founded ordering with a smallest element 0 such that $|A|=|B|$ if $A$ and $B$ are variants.

The following auxiliary notion will be used below.

Definition 3.3. $C^{\prime}$ is called a head instance of a clause $C$ if $C^{\prime}=C \theta$ for some substitution that instantiates only variables of $C$ that appear in its head.

First we provide a method for proving (strong) termination of Prolog programs in homogeneous form. Our key concept is the following one.

Definition 3.4. A homogeneous Prolog program $P$ is called acceptable w.r.t. a level mapping $\|$ and a $\Theta$-model $I$ of $P$ if for all head instances $A \leftarrow B_{1}, \ldots, B_{n}$ of a clause of $P$ the following implication holds for $i \in[1, n]$ :

$$
\text { if } \left.I \models\left\langle B_{1}, \ldots, B_{i-1}, \eta\right\rangle \text { then }|A|\right\rangle\left|B_{i} \eta\right| \text {. }
$$


$P$ is called acceptable if it is acceptable w.r.t. some level mapping and a $\Theta$-model of $P$.

The relevance of the notion of acceptability is clarified by the following theorem.

Theorem 3.5. (Soundness III) Let $P$ be a homogeneous Prolog program. If $P$ is acceptable then it strongly terminates.

The following notion will be useful in the proof.

Definition 3.6. Consider an LD-derivation $\xi$. Let $G$ be a goal in $\xi$. Let $k$ be the minimum length of a goal in the suffix of $\xi$ starting at $G$ and let $H$ be the first goal in this suffix with length $k$. We call $H$ the shortest goal of $\xi$ under $G$.

Proof of Theorem 3.5. Suppose by contradiction that there exists an infinite LDderivation of $P \cup\{G\}$. Call it $\xi$. Denote $G$ by $H_{0}$. We first define two infinite sequences $G_{1}, G_{2}, \ldots$ and $H_{1}, H_{2}, \ldots$ of goals of $\xi$ by the following formula for $j \geqq 1$ :

$G_{j}$ is the shortest goal of $\xi$ under $H_{j-1}$,

$H_{j}$ is the direct descendant of $G_{j}$ in $\xi$.

Fix $j \geqq 1$. Let $A \leftarrow B_{1}, \ldots, B_{n}$ be the input clause and $\theta$ the mgu used to obtain $H_{j}$ from $G_{j}$. By the choice of $G_{j}$ and $H_{j}$ we have $l\left(G_{j}\right) \leqq l\left(H_{j}\right)$, so $n \geqq 1$. $G_{j}$ is of the form $\leftarrow C_{1}, \ldots, C_{k}$ where $k \geqq 1$ and $H_{j}$ is of the form $\leftarrow\left(B_{1}, \ldots, B_{n}, C_{2}, \ldots, C_{k}\right) \theta$. By definition, no goal of $\xi$ under $G_{j}$ is of length less than $k$, so $G_{j+1}$ is of the form $\leftarrow\left(B_{i}, \ldots, B_{n}, C_{2}, \ldots, C_{k}\right) \theta \eta$ for some $\eta$, where $i \in[1, n-1]$. This means that there exists an LD-refutation of $P \cup\left\{\leftarrow\left(B_{1}, \ldots, B_{i-1}\right) \theta\right\}$ with c.a.s. $\eta$. This refutation is obtained by deleting from all goals of $\xi$ between and including $H_{j}$ and $G_{j+1}$ all occurrences of the instantiated versions of $B_{i} \theta, \ldots, B_{n} \theta, C_{2} \theta, \ldots, C_{n} \theta$.

By the Soundness Theorem 2.11 we have $I \models\left\langle\left(B_{1}, \ldots, B_{i-1}\right) \theta, \eta\right\rangle$. By the acceptability of $P$

$$
|A \theta|>\left|B_{i} \theta \eta\right| .
$$

By Assumption 2.10 the mgu $\mu$ used to obtain $H_{j+1}$ from $G_{j+1}$ does not bind the variables of the selected atom $B_{i} \theta \eta$. So $B_{i} \theta \eta=B_{i} \theta \eta \mu$ and consequently

$$
\left|B_{i} \theta \eta\right|=\left|B_{i} \theta \eta \mu\right| \text {. }
$$

Thus assuming $j>1$, we have

$$
\left|C_{1}\right|=\left|C_{1} \theta\right|,
$$

$\left(C_{1}\right.$ is the first atom of $G_{j}$ and $B_{i} \theta \eta$ is the first atom of $\left.G_{j+1}\right)$. But $\theta$ unifies $A$ and $C_{1}$, so

$$
\left|C_{1} \theta\right|=|A \theta| .
$$

By (16), (18), and (19) we conclude, assuming $j>1$,

$$
\left|C_{1}\right|>\left|B_{i} \theta \eta\right| \text {. }
$$

Thus applying the level mapping | | to the first atoms of the goals $G_{2}, G_{3}, \ldots$ we obtain an infinite descending sequence of elements of a well-founded ordering. This yields a contradiction.

We now prove a converse of the Soundness III Theorem 3.5.

For a Prolog program $P$ that strongly terminates and a goal $G$, denote by 
$\operatorname{nodes}_{P}(G)$ the number of nodes in the LD-tree of $P \cup\{G\}$. The following lemma summarizes the relevant properties of $\operatorname{nodes}_{P}(G)$.

Lemma 3.7. (LD-tree) Let $P$ be a Prolog program that strongly terminates. Then

(i) $\operatorname{nodes}_{P}(G)=\operatorname{nodes}_{P}(H)$ if $G$ and $H$ are variants,

(ii) $\operatorname{nodes}_{P}(H)<\operatorname{nodes}_{P}(G)$ for all non-root nodes $H$ in the $L D$-tree of $P \cup\{G\}$,

(iii) $\operatorname{nodes}_{P}(H) \leqq \operatorname{nodes}_{P}(G)$ for all prefixes $H$ of $G$.

Proof. (i) By a simple generalization of the Variant Lemma 2.8 of Apt [1] to the class of Prolog programs, an isomorphism between the LD-trees of $P \cup\{G\}$ and $P \cup\{H\}$ can be established. (ii), (iii) Immediate by the definition.

We are now in position to prove the desired result.

Theorem 3.8. (Completeness III) Let $\boldsymbol{P}$ be a homogeneous Prolog program. Suppose that $P$ strongly terminates. Then $P$ is acceptable.

Proof. Put for an atom $A$

$$
|A|=\operatorname{nodes}_{P}(\leftarrow A) .
$$

By Lemma 3.7 (i) || is a level mapping. We now prove that $P$ is acceptable w.r.t. | | and $N_{P}$, the least $\Theta$-model of $P$. To this end consider a clause $C$ with head $A_{0}$ and its head instance $C \theta=A \leftarrow B_{1}, \ldots, B_{n}$ where $\operatorname{Dom}(\theta) \subseteq \operatorname{Var}\left(A_{0}\right)$. Let us assume that $C \theta$ is disjoint with $C$. Then $A$ is disjoint with $A_{0}, A=A_{0} \theta$ and $\operatorname{Dom}(\theta) \subseteq$ $\operatorname{Var}\left(A_{0}\right)$, so $\theta$ is idempotent and $A \theta=A$. Thus $\theta$ unifies $A$ and $A_{0}$ and it is easy to see that in fact $\theta$ is an $m g u$ of $A$ and $A_{0}$. Thus $\leftarrow B_{1}, \ldots, B_{n}$ is a resolvent of $\leftarrow A$ with the input clause $C$. By Lemma 3.7 (ii)

$$
\operatorname{nodes}_{P}(\leftarrow A)>\operatorname{nodes}_{P}\left(\leftarrow B_{1}, \ldots, B_{n}\right) \text {. }
$$

This conclusion was reached under the assumption that $C \theta$ is disjoint with $C$ but Lemma 3.7 (i) allows us to dispense us with this assumption. Suppose now that $N_{P} \models\left\langle B_{1}, \ldots, B_{i-1}, \eta\right\rangle$ for some $i \in[1, n]$ and substitution $\eta$. Then by the Completeness Theorem 2.24 there exists an LD-refutation of $\leftarrow B_{1}, \ldots, B_{i-1}$ with c.a.s. $\eta$, so $\leftarrow\left(B_{i}, \ldots, B_{n}\right) \eta$ is a node in the LD-tree of $P \cup\left\{\leftarrow B_{1}, \ldots, B_{n}\right\}$. By Lemma 3.7 (ii)

$$
\operatorname{nodes}_{P}\left(\leftarrow B_{1}, \ldots, B_{n}\right) \geqq \operatorname{nodes}_{P}\left(\leftarrow\left(B_{i}, \ldots, B_{n}\right) \eta\right)
$$

and by Lemma 3.7 (iii)

$$
\operatorname{nodes}_{P}\left(\leftarrow\left(B_{i}, \ldots, B_{n}\right) \eta\right) \geqq \operatorname{nodes}_{P}\left(\leftarrow B_{i} \eta\right) .
$$

By (20), (21), and (22) we now conclude

$$
\operatorname{nodes}_{P}(\leftarrow A)>\operatorname{nodes}_{P}\left(\leftarrow B_{i} \eta\right),
$$

i.e. $|A|>\left|B_{i} \eta\right|$.

This shows that $P$ is acceptable.

Thus we proved an equivalence between the notions of acceptability and strong termination for homogeneous Prolog programs.

Now, every Prolog program can be easily transformed into a homogeneous program with the same termination behaviour. 
Theorem 3.9. Let $P$ be a Prolog program and $G$ a goal. Then the $L D$-tree of $P \cup\{G\}$ is finite iff the $L D$-tree of $\operatorname{Hom}(P) \cup\{G\}$ is finite.

The following lemma is useful.

Lemma 3.10. Let $G$ be a goal and $C$ a clause. $G$ and $C$ have LD-resolvent $\leftarrow \mathbf{Q} \theta$ with mgu $\theta$ iff $G$ and $\operatorname{Hom}(C)$ have resolvent $\leftarrow x_{1} \alpha=t_{1}, \ldots, x_{n} \alpha=t_{n}, Q$ with $m g u \alpha$ and $\theta$ is the c.a.s. of $\leftarrow x_{1} \alpha=t_{1}, \ldots, x_{n} \alpha=t_{n}$, where $t_{1}, \ldots, t_{n}\left(\right.$ resp. $\left.x_{1}, \ldots, x_{n}\right)$ are the arguments of the head of $C(\operatorname{resp} . \operatorname{Hom}(C))$.

Proof. See [4].

\section{Proof of Theorem 3.9}

The LD-trees (in $P$ and in $H o m(P)$ ) are finitely branching, so by König Lemma it suffices to show that $G$ has an infinite derivation in $P$ iff $G$ has an infinite derivation in $\operatorname{Hom}(P)$. The result follows by Lemma 3.10.

Corollary 3.11. Let $P$ be a Prolog program. Then $P$ strongly terminates iff $\operatorname{Hom}(P)$ strongly terminates.

This allows us to reason about termination of Prolog programs by transforming them first to a homogeneous form and then using the notion of acceptability. We offer now an alternative, direct way of reasoning about termination. To this end the following auxiliary notion will be needed.

Definition 3.12. Let $P$ be a Prolog program and | | a level mapping. An atom $A$ is called stable w.r.t. || if $|A| \geqq|A \theta|$ for every $m g u \theta$ of $A$ and a disjoint with $A$ variant of a head of a non-unit clause of $P$.

Intuitively, an atom $A$ is stable w.r.t. a level mapping || if $A$ is sufficiently instantiated so that the value of || on every instance of $A$ can be defined by means of the arguments of $A$. Note that atoms with built-in relations are automatically stable w.r.t. every level mapping.

The following is a generalization of Definition 3.4 to arbitrary Prolog programs.

Definition 3.13. A Prolog program $P$ is called acceptable w.r.t. a level mapping || and a $\Theta$-model $I$ of $P$ if for all head instances $A \leftarrow B_{1}, \ldots, B_{n}$ of a clause of $P$ the following implication holds for $i \in[1, n]$ :

if $I \models\left\langle B_{1}, \ldots, B_{i-1}, \eta\right\rangle$ then

(i) $|A|>\left|B_{i} \eta\right|$,

(ii) $B_{i} \eta$ is stable w.r.t. $\mid$.

$P$ is called acceptable if it is acceptable w.r.t. some level mapping and a $\Theta$-model of $P$.

It is important to note the following.

Lemma 3.14. Let $P$ be a homogeneous Prolog program and || a level mapping. Then every atom is stable w.r.t. $\mid$.

Proof. Suppose an atom $A$ unifies with a disjoint with $A$ variant $B$ of a head of a non-unit clause of $P$. $B$ is an elementary atom, so $A$ is an instance of $B$, say $A=B \eta$ with $\eta$ such that $\operatorname{Dom}(\eta)=\operatorname{Var}(B)$. Then $A \eta=A$, so $\eta$ unifies $A$ and $B$. 
Let now $\theta$ be an $m g u$ of $A$ and $B$. Then $A \theta$ is more general than $A \eta$, i.e. $A \theta$ is more general than $A$. Also $A$ is more general than $A \theta$, so $A$ and $A \theta$ are variants and consequently $|A|=|A \theta|$.

Corollary 3.15. For homogeneous programs both definitions of acceptability coincide.

The following theorem is a generalization of the Soundness III Theorem 3.5.

Theorem 3.16. (Soundness IV) Let $P$ be a Prolog program. Suppose $P$ is acceptable. Then $P$ strongly terminates.

Proof. The proof is completely analogous to that of the Soundness III Theorem 3.5. The only difference is that instead of (17) we can now only claim by condition (ii) of acceptability

$$
\left|B_{i} \theta \eta\right| \geqq\left|B_{i} \theta \eta \mu\right|,
$$

so assuming $j>1$ we now only have

$$
\left|C_{1}\right| \geqq\left|C_{1} \theta\right| \text {. }
$$

instead of (18). However, this weaker conclusion is still sufficient to yield the same contradiction as in the proof of Theorem 3.5.

Ideally, we would like to prove the converse of the Soundness IV Theorem 3.16, that is Prolog programs that strongly terminate are acceptable. Unfortunately this is not the case.

Theorem 3.17. There exists a Prolog program $P$ that strongly terminates but is not acceptable.

Proof. Consider the following program $P$ :

$$
\begin{aligned}
& p(f(X)) \leftarrow \text { nonvar }(X), p(X) . \\
& p(f(f(X))) \leftarrow \text { nonvar }(X), p(X) .
\end{aligned}
$$

It is easy to see that all LD-derivations of $P$ terminate. In fact, in every LDderivation of $P$ a goal of the form $\leftarrow p(y)$ leads to a failure in two steps and a goal of the form $\leftarrow p\left(f^{n}(y)\right)$, where $n \geqq 1$, leads to a goal of the form $\leftarrow p\left(f^{k}(y)\right)$, where $k<n$, in two steps.

Suppose now that $P$ is acceptable w.r.t. some level mapping || and a $\Theta$-model $I$. Then due to condition (i)

$$
|p(f(f(Y)))|>|p(f(Y))|
$$

because nonvar $(f(Y))$ holds. Also $p(f(Y))$ is stable w.r.t. | |, so

$$
|p(f(Y))| \geqq|p(f(f(X)))|
$$

which gives a contradiction.

It may seem disappointing that we opted here for a notion of acceptability that did not allow us to prove its equivalence with strong termination for all Prolog programs. Clearly, it is possible to characterize strong termination by means of well-founded relations for all Prolog programs. To this end it suffices to use the concept of a level mapping defined on goals, with the condition that $|H|<|G|$ whenever $H$ is a direct descendant of $G$ in an LD-derivation. However, such a 
characterization of strong termination is hardly of any use when proving termination because it requires an analysis of arbitrary goals. In contrast, the definition of acceptability refers only to the program clauses and calls for the use of a level mapping defined only on atoms, so it is simpler to use.

On the other hand, the introduction of homogeneous programs allows us to draw the following conclusion.

Theorem 3.18. Let $P$ be a Prolog program. Then $P$ strongly terminates iff $\operatorname{Hom}(P)$ is acceptable.

Proof. By the Soundness III Theorem 3.5 and Completeness III Theorem 3.8 applied to $\operatorname{Hom}(P)$, and Corollary 3.11 .

\section{Applications}

We illustrate the use of the results established in the previous section to prove strong termination of some Prolog programs. We start by considering the program list given in Section 1.

Then we show how a relation that strongly terminates can be treated as a built-in relation when proving strong termination of a program depending on this relation. This allows us to prove strong termination in a modular way. We illustrate this method by proving strong termination of two well-known Prolog programs.

First, we define by structural induction the function | | on terms by putting:

$$
\begin{aligned}
& |x|=0 \text { if } x \text { is a variable, } \\
& \left|f\left(x_{1}, \ldots, x_{n}\right)\right|=0 \text { if } f \neq[\cdot \mid \cdot], \\
& |[x \mid x s]|=|x s|+1 .
\end{aligned}
$$

It is useful to note that for a list $x s,|x s|$ equals its length. This function will be used in the examples below.

\section{List}

Consider the program list from Sect. 1:

$\left(l_{1}\right)$ list $([]) \leftarrow$.

$\left(l_{2}\right) \quad$ list $([\mathrm{X} \mid \mathrm{Xs}]) \leftarrow$ nonvar (Xs), list (Xs).

To prove that list strongly terminates we show that it is acceptable. We define a level mapping | | by putting

$$
\begin{aligned}
& |\operatorname{list}(x s)|=|x s| \\
& \mid \text { nonvar }(x s) \mid=0 .
\end{aligned}
$$

Clearly, $|A|=|B|$ if $A$ and $B$ are variants, so || is indeed a level mapping. Next, we take the $\Theta$-base $\Theta_{P}$ as the $\Theta$-model of list.

Theorem 4.1. list is acceptable w.r.t. $\mid$ and $\Theta_{P}$.

Proof. Consider a head instance $C=A \leftarrow B_{1}, B_{2}$ of $\left(l_{2}\right)$. It is of the form

$$
\text { list }([x \mid x s]) \leftarrow \text { nonvar }(x s) \text {, list }(x s) \text {. }
$$


Claim 1. $|A|>\left|B_{1} \eta\right|$.

Proof. Note that $\mid$ list $([x \mid x S])|>0=|$ nonvar $(x s \eta) \mid$.

Suppose now $\Theta_{p} \vDash\left\langle B_{1}, \eta\right\rangle$. Then $\eta=\varepsilon$ and $B_{1} \eta=$ nonvar $(x s)$ with $x s \notin V a r$.

Claim 2. $|A|>\left|B_{2} \eta\right|$.

Proof. Note that $|A|=|\operatorname{list}([x \mid x s])|=|[x \mid x s]|>|x s|=\mid$ list $(x s)|=| B_{2} \eta \mid$.

Claim 3. $B_{2} \eta$ is stable w.r.t. $\mid$.

Proof. Suppose $B_{2} \eta$ unifies with a variant list $\left(\left[x^{\prime} \mid x s^{\prime}\right]\right)$ of the head of the clause $\left(l_{2}\right)$. Since $x_{s} \notin \operatorname{Var}, B_{2} \eta$ is an instance of list $\left(\left[x^{\prime} \mid x s^{\prime}\right]\right)$. As in the proof of Lemma 3.14 this implies that for any mgu $\theta$ of $B_{2} \eta$ and list $\left(\left[x^{\prime} \mid x s^{\prime}\right]\right)$ we have $\left|B_{2} \eta\right|=\left|B_{2} \eta \theta\right|$.

\section{Modularity}

In the proof of Theorem 3.5 the level mapping of built-in relations is not used. This is due to the fact that the built-in relations always terminate and never occur in the head of a clause. So we can assume that $|A|=0$ if $A$ is a built-in atom.

This observation provides an idea of how to prove the strong termination of a Prolog program in a modular way. Before formalizing this idea we show how the relation list previously defined can be treated as a built-in in the proof of the strong termination of a Prolog program.

Example 4.2. Consider the following program APPEND:

$$
\begin{aligned}
&\left(a_{1}\right) \quad \mathrm{a}([], Y \mathrm{~s}, Y \mathrm{~s}) \leftarrow \\
& \text { list }(Y \mathrm{~s}) . \\
&\left(a_{2}\right) \quad \mathrm{a}([\mathrm{X} \mid \mathrm{Xs}], Y \mathrm{~s},[\mathrm{X} \mid \mathrm{Zs}]) \leftarrow \\
& \text { nonvar }(\mathrm{Xs}), \mathrm{a}(\mathrm{Xs}, \mathrm{Ys}, \mathrm{Zs}) .
\end{aligned}
$$

augmented by the clauses $\left(l_{1}\right)$ and $\left(l_{2}\right)$ defining the list program.

To prove that APPEND strongly terminates we regard APPEND as union of the program append, containing only the clauses $\left(a_{1}\right)$ and $\left(a_{2}\right)$ of APPEND, with the program list. In append the relation list does not occur in the head of any clause. We already proved that list strongly terminates. Thus the relation list can be treated as a built-in with the semantics given by an arbitrary $\Theta$-model $I_{0}$ of the program list. Hence, to show that APPEND strongly terminates, it is sufficient to prove that append is acceptable w.r.t. a model of APPEND and a level mapping || s.t. $|A|$ is 0 if $A$ is a built-in or is an atom of the form list $\left(x_{s}\right)$. We choose the following level mapping:

$$
\begin{aligned}
& |a(x, y, z)|=|x|, \\
& |A|=0 \text { if } A \text { is a built-in or list (xs). }
\end{aligned}
$$

Next, we define a $\Theta$-interpretation for the relation $a$ by putting

$$
I=\{\langle a(x s, y s, z s), \eta\rangle|\quad| x s \eta|+| y s \eta|=| z s \eta \mid\} .
$$

Lemma 4.3. $I \cup I_{0}$ is a $\Theta$-model of APPEND.

Proof. Clearly $I \cup I_{0}$ is a model of list. 
Let $A=a(r, s, t)$ and let $a\left([], Y_{s}^{\prime}, Y_{s}^{\prime}\right) \leftarrow \operatorname{list}\left(Y_{s}^{\prime}\right)$ be a variant of $\left(a_{1}\right)$ disjoint with $A$. Suppose that $\theta=\operatorname{mgu}\left(A, a\left([], Y_{s}^{\prime}, Y_{s}^{\prime}\right)\right)$ exists and suppose that $I \cup I_{0} \vDash\left\langle\operatorname{list}\left(Y_{s}^{\prime}\right) \theta, \eta\right\rangle$, with $\eta$ satisfying the restriction of Definition 2.28. We have to show that $I \cup I_{0}=$ $\langle A,(\theta \eta) \mid A\rangle$. We have that $r \theta=[], s \theta=t \theta=Y_{s}^{\prime} \theta$. Then $|r \theta|+|s \theta|=|t \theta|$ and so $|r \theta \eta|+|s \theta \eta|=|t \theta \eta|$. Hence $I \cup I_{0} \models\langle A,(\theta \eta) \mid A\rangle$ holds.

Let now $a\left(\left[X^{\prime} \mid X_{s}^{\prime}\right], Y_{s}^{\prime},\left[X^{\prime} \mid Z_{s}^{\prime}\right]\right) \leftarrow \operatorname{nonvar}\left(X_{s}^{\prime}\right), a\left(X_{s}^{\prime}, Y_{s}^{\prime}, Z_{s}^{\prime}\right)$ be a variant of $\left(a_{2}\right)$ disjoint with $A$. Suppose that $\theta=m g u\left(A, a\left(\left[X^{\prime} \mid X_{s}^{\prime}\right], Y_{s}^{\prime},\left[X^{\prime} \mid Z_{s}^{\prime}\right]\right)\right)$ exists and suppose that $I \cup I_{0} \vDash\left\langle\left(\operatorname{nonvar}\left(X_{s}^{\prime}\right), a\left(X_{s}^{\prime}, Y_{s}^{\prime}, Z_{s}^{\prime}\right)\right) \theta, \eta\right\rangle$, with $\eta$ satisfying the restriction of Definition 2.28. We have to show that $I \cup I_{0} \models\langle A,(\theta \eta) \mid A\rangle$. Clearly (nonvar $\left.\left(X_{s}^{\prime}\right) \theta, a\left(X_{s}^{\prime}, Y_{s}^{\prime}, Z_{s}^{\prime}\right) \theta, \varepsilon, \eta\right)$ is a good tuple. Then, by the semantics of nonvar, it follows that $I \cup I_{0} \vDash\left\langle\left(\right.\right.$ nonvar $\left.\left.\left(X_{s}^{\prime}\right), a\left(X_{s}^{\prime}, Y_{s}^{\prime}, Z_{s}^{\prime}\right)\right) \theta, \eta\right\rangle$ iff $I \cup I_{0} \models\left\langle a\left(X_{s}^{\prime}, Y_{s}^{\prime}, Z_{s}^{\prime}\right) \theta, \eta\right\rangle$, with $X_{s}^{\prime} \theta \notin$ Var. Then we have $\left|X_{s}^{\prime} \theta \eta\right|+\left|Y_{s}^{\prime} \theta \eta\right|=\left|Z_{s}^{\prime} \theta \eta\right|$ and, by $r \theta=\left[X^{\prime} \mid X_{s}^{\prime}\right] \theta$, $s \theta=Y_{s} \theta$ and $t \theta=\left[X^{\prime} \mid Z_{s}^{\prime}\right] \theta$ it follows that $|r \theta \eta|+|s \theta \eta|=|t \theta \eta|$. Hence $I \cup I_{0} \models$ $\langle A,(\theta \eta) \mid A\rangle$ holds.

This concludes the proof that $I \cup I_{0}$ is a $\Theta$-model of APPEND.

Theorem 4.4. append is acceptable w.r.t. | | and $I \cup I_{0}$.

Proof. Analogous to that of Theorem 4.1, due to the similarity between clauses $\left(a_{2}\right)$ and $\left(l_{2}\right)$.

We can now formulate our modular approach to termination.

Definition 4.5. Let $P_{1}$ and $P_{2}$ be two Prolog programs. We say that $P_{2}$ extends $P_{1}$, and write $P_{1}<P_{2}$, if

(i) $P_{1}$ and $P_{2}$ define different relations,

(ii) no relation defined in $P_{2}$ occurs in $P_{1}$.

Informally, $P_{2}$ extends $P_{1}$ if $P_{2}$ defines new relations, possibly using the relations defined already in $P_{1}$. For example the program APPEND extends the program list.

The following theorem formalizes the idea used to prove termination of the APPEND program.

Theorem 4.6. (Modularity) Suppose $P_{2}$ extends $P_{1}$. Assume that

(i) $P_{1}$ is acceptable,

(ii) $P_{2}$ is acceptable w.r.t. a $\Theta$-model I of $P_{1} \cup P_{2}$ and a level mapping || such that $|A|=0$ if $A$ contains a relation defined in $P_{1}$.

Then $P_{1} \cup P_{2}$ strongly terminates.

Proof. $P_{2}$ extends $P_{1}$. Thus $P_{1} \cup P_{2}$ strongly terminates iff $P_{1}$ strongly terminates and $P_{2}$ strongly terminates when the relations defined in $P_{1}$ are treated as built-in's defined by

$\llbracket p \rrbracket=\left\{\langle A, \eta\rangle \mid A\right.$ contains $p$ and there exists an LD-refutation of $P_{1} \cup\{\leftarrow A\}$ with c.a.s. $\eta\}$.

Now, by (i) and the Soundness IV Theorem 3.16 $P_{1}$ strongly terminates. To deal with the other conjunct consider $N_{P_{1} \cup P_{2}}$, the least $\Theta$-model of $P_{1} \cup P_{2}$. By (ii) and Corollary $2.13 P_{2}$ is acceptable w.r.t. $N_{P_{1} \cup P_{2}}$ and the level mapping | |. Moreover, by Corollary 2.25 and the fact that $P_{2}$ extends $P_{1}$ we have for all atoms $A$ containing a relation $p$ defined in $P_{1}$

$$
N_{P_{1} \cup P_{2}} \models\langle A, \eta\rangle \text { iff }\langle A, \eta\rangle \in \llbracket p \rrbracket .
$$


Thus by the Soundness IV Theorem $3.16 P_{2}$ strongly terminates when the relations defined in $P_{1}$ are treated as built-in's defined as above.

This concludes the proof of the theorem.

We illustrate the use of this theorem in the example below.

\section{Unification}

Consider the program UNIFY (for unification without occurs check) from Sterling and Shapiro [page 150] [17]. In this program several built-in's, namely var, nonvar, $=$, constant, compound, functor, $>$ are used. The meaning of them was already given in Section 2. Additionally, the function "-" (minus) is used on terms. Its meaning is implicitly referred within the description of the meaning of ": $=$ ". For instance, $\langle x:=3-1,\{x / 2\}\rangle \in \mathbb{I}:=\mathbb{\rrbracket}$.

The program UNIFY consists of the following clauses.

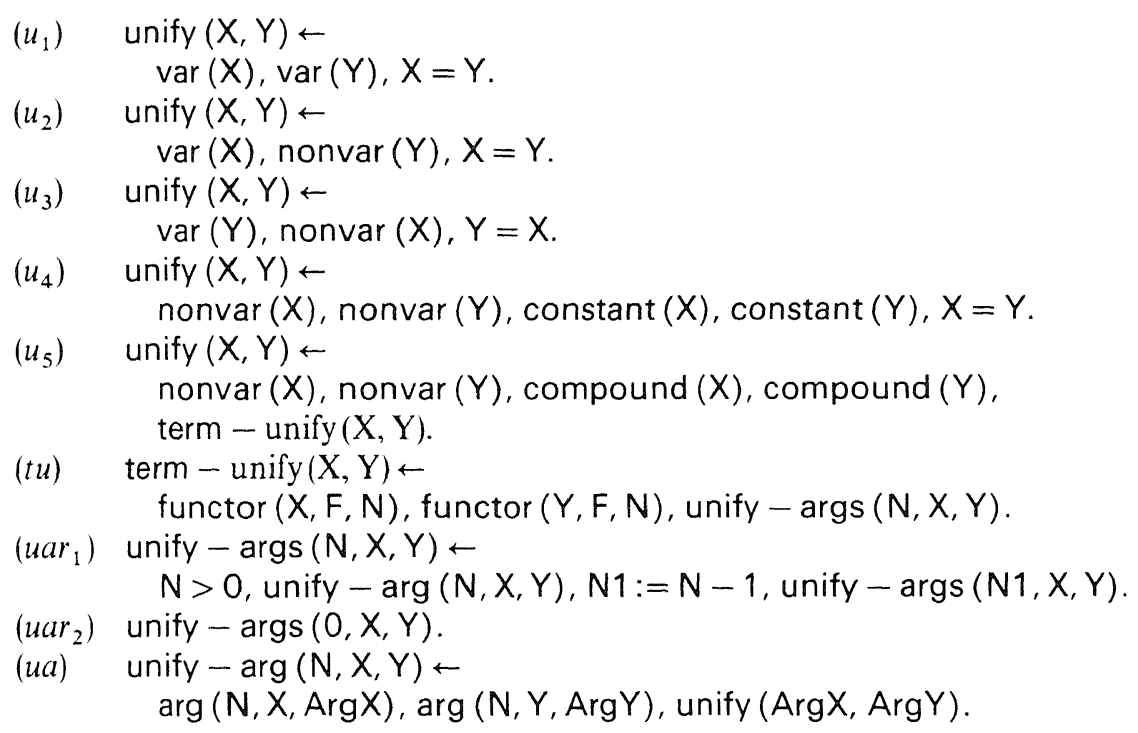

We assume that UNIFY operates on the domain of natural numbers over which the built-in relation $>$ and the function - , both written in infix notation, are defined.

In Pieramico [15] it was proved that UNIFY terminates for ground goals by showing that the program obtained by deleting all built-in relations is acceptable in the sense of Apt and Pedreschi [5].

We prove here a stronger statement, namely that UNIFY strongly terminates by showing that it is acceptable in the sense of Definition 3.13.

For the subsequent analysis it is important to understand how this program operates. Intuitively, the goal $\leftarrow$ unify $(\mathrm{s}, \mathrm{t})$ yields an mgu of $s$ and $t$ as a computed answer substitution if $s$ and $t$ unify, and otherwise it fails. It is evaluated as follows. If either $s$ or $t$ is a variable, then the built-in relation = is called (clauses $\left(u_{1}\right)-\left(u_{3}\right)$ ). It assigns to the term out of $s, t$ which is a variable the other term. If both $s$ and $t$ are variables (clause $\left.\left(u_{1}\right)\right)$ then $s$ is chosen. If neither $s$ nor $t$ is a variable, but both are constants, then it is tested - again by means of $=-$ whether they are equal 
(clause $\left(u_{4}\right)$ ). The case when both $s$ and $t$ are compound terms is handled in clause $\left(u_{5}\right)$ by calling the relation term - unify. This relation is defined by clause $(t u)$.

The goal $\leftarrow$ term - unify $(\mathrm{s}, \mathrm{t})$ is evaluated by first identifying the form of $s$ and $t$ by means of the built-in relation functor. If for some function symbol $f$ and $n \geqq 0$, the term $s$ is of the form $f\left(s_{1}, \ldots, s_{n}\right)$ and the term $t$ is of the form $f\left(t_{1}, \ldots, t_{n}\right)$, then the relation unify - args is called. This relation is defined by clauses $\left(u a r_{1}\right)$ and $\left(\right.$ uar $\left._{2}\right)$.

The goal $\leftarrow$ unify - args $(n, s, t)$ succeeds if the sequence of the first $n$ arguments of $s$ can be unified with the sequence of the first $n$ arguments of $t$. When $n>0$, clause $\left(u_{a r}\right)$ is used and these arguments are unified pairwise starting with the last pair. This last pair is dealt with by calling the relation unify - arg which is defined by clause $(u a)$.

The goal $\leftarrow$ unify - $\arg (n, s, t)$ is evaluated by first extracting the $n$-th arguments of $s$ and $t$ by means of the built-in relation arg, and then calling unify recursively on these arguments. If this call succeeds, the produced c.a.s. modifies $s$ and $t$, and the recursive call of unify - args in clause $\left(u a r_{1}\right)$ operates on this modified pair of $s$ and $t$. Finally, when $n=0$, unify - args $(n, s, t)$ succeeds immediately (clause $\left(\right.$ uar $\left._{2}\right)$ ). It is clear from this description what is the intended meaning of the defined relations unify, term - unify, unify - args and unify - arg. In the proof of the strong termination of UNIFY only partial information about the meaning of these relations is needed. This information is captured in the $\Theta$-model $I$ we use.

Let us first define a level mapping | |. To this end we use the lexicographic ordering $<$ defined on triples of natural numbers. In this ordering $\left\langle n_{1}, n_{2}, n_{3}\right\rangle<$ $\left\langle m_{1}, m_{2}, m_{3}\right\rangle$ iff $\left(n_{1}<m_{1}\right)$ or $\left(n_{1}=m_{1} \wedge n_{2}<m_{2}\right)$ or $\left(n_{1}=m_{1} \wedge n_{2}=m_{2} \wedge n_{3}<m_{3}\right)$.

For brevity we write $\operatorname{Var}(s, t)$ instead of $\operatorname{Var}(s) \cup \operatorname{Var}(t)$. We put

$$
\begin{aligned}
& \mid \text { unify }(s, t) \mid=\langle\operatorname{card}(\operatorname{Var}(s, t)), \operatorname{nodes}(s)+\operatorname{nodes}(t), 1\rangle, \\
& |\operatorname{term}-\operatorname{unify}(s, t)|=\langle\operatorname{card}(\operatorname{Var}(s, t)), \operatorname{nodes}(s)+\operatorname{nodes}(t), 0\rangle, \\
& \mid \text { unify }-\operatorname{args}(n, s, t) \mid=\langle\operatorname{card}(\operatorname{Var}(s, t)), f(n, s, t), 3\rangle, \\
& \mid \text { unify- } \arg (n, s, t) \mid=\left\langle\operatorname{card}(\operatorname{Var}(s, t)), \operatorname{nodes}\left(s_{n}\right)+\operatorname{nodes}\left(t_{n}\right), 2\right\rangle, \\
& |A|=\langle 0,0,0\rangle \text { if } A \text { built-in, }
\end{aligned}
$$

where $\operatorname{card}(S)$ indicates the cardinality of the set $S$ and $f(n, s, t)$ denotes the sum of the number of nodes of the $i$-th component of $s$ and $t$ for $i \in[1, n]$, that is

$$
f(n, s, t)=\sum_{i=1}^{n}\left(\operatorname{nodes}\left(s_{i}\right)+\operatorname{nodes}\left(t_{i}\right)\right) \text {. }
$$

Next we define the $\Theta$-interpretation $I$.

$$
\begin{aligned}
I= & \{\langle\text { unify }(s, t), \theta\rangle,\langle\text { term }- \text { unify }(s, t), \theta\rangle \mid \operatorname{Inv}(s, t, \theta)\} \cup \\
& \{\langle\text { unify }-\operatorname{args}(n, s, t), \theta\rangle,\langle\text { unify }-\arg (n, s, t), \theta\rangle \mid n \text { natural number, } \operatorname{Inv}(s, t, \theta))\},
\end{aligned}
$$

where $\operatorname{Inv}(s, t, \theta)$ is the assertion below:

$$
\begin{aligned}
\operatorname{Ran}(\theta) & \cong \operatorname{Var}(s, t) \wedge(\operatorname{Var}(s \theta, t \theta)=\operatorname{Var}(s, t) \\
& \Rightarrow \operatorname{nodes}(s)+\operatorname{nodes}(t)=\operatorname{nodes}(s \theta)+\operatorname{nodes}(t \theta)) .
\end{aligned}
$$

The following example clarifies the $\Theta$-interpretation and level mapping we have chosen. Consider the goal $G_{1}=\leftarrow$ unify $(f(s), f(t))$.

1) $G_{1}$ calls $G_{2}=\leftarrow$ term - unify $(f(s), f(t))$ using clause $\left(u_{5}\right)$; 
2) $G_{2}$ calls $G_{3}=\leftarrow$ unify $-\operatorname{args}(1, f(s), f(t))$ using clause $(t u)$;

3) $G_{3}$ calls $G_{4}=\leftarrow$ unify $-\arg (1, f(s), f(t))$ and $G_{5}=\leftarrow$ unify $-\operatorname{args}(0, f(s) \theta, f(t) \theta)$ using clause $\left(u a r_{1}\right)$, where $\theta$ is a c.a.s. of $G_{4}$;

4) $G_{4}$ calls $G_{6}=\leftarrow u n i f y(s, t)$ using clause $(u a)$.

Let $\mid$ unify $(f(s), f(t)) \mid=\left\langle m_{1}, m_{2}, 1\right\rangle$,

$$
\begin{aligned}
& \mid \text { unify }-\operatorname{args}(1, f(s), f(t)) \mid=\left\langle k_{1}, k_{2}, 3\right\rangle, \\
& \mid \text { unify- } \operatorname{args}(1, f(s) \theta, f(t) \theta) \mid=\left\langle k_{1}^{\prime}, k_{2}^{\prime}, 3\right\rangle \text {. Then } \\
& \mid \text { term-unify }(f(s), f(t)) \mid=\left\langle m_{1}, m_{2}, 0\right\rangle, \\
& \mid \text { unify }-\arg (1, f(s), f(t)) \mid=\left\langle k_{1}, k_{2}, 2\right\rangle \text { and } \\
& \mid \text { unify }(s, t) \mid=\left\langle k_{1}, k_{2}, 1\right\rangle .
\end{aligned}
$$

We now show that when $G_{i}$ calls $G_{j}, i, j \in[1,6],\left|G_{i}\right|>\left|G_{j}\right|$.

For 1) we need $\mid$ unify $(f(s), f(t))|>|$ term - unify $(f(s), f(t)) \mid$, which holds because $1>0$.

For 2) we need $\mid$ term - unify $(f(s), f(t))|>|$ unify - args $(1, f(s), f(t)) \mid$, which is satisfied because $m_{1}=k_{1}$ and $m_{2}>k_{2}$.

For 3) we need $\mid$ unify-args $(1, f(s), f(t))|>|$ unify-arg $(1, f(s), f(t)) \mid$, which is satisfied because $3>2$, and $\mid$ unify $-\operatorname{args}(1, f(s), f(t))|>|$ unify $-\operatorname{args}(0, f(s) \theta, f(t) \theta) \mid$, which is satisfied when $k_{1}>k_{1}^{\prime}$, or $k_{1}=k_{1}^{\prime}$ and $k_{2}=k_{2}^{\prime}$. These conditions are satisfied if

$$
\begin{aligned}
& \operatorname{Ran}(\theta) \subseteq \operatorname{Var}(f(s), f(t)) \text { and } \\
& \operatorname{Var}(f(s) \theta, f(t) \theta)=\operatorname{Var}(f(s), f(t)) \Rightarrow \operatorname{nodes}(f(s))+\operatorname{nodes}(f(t)) \\
& \quad=\operatorname{nodes}(f(s) \theta)+\operatorname{nodes}(f(t) \theta), \\
& \text { i.e. if } \operatorname{Inv}(f(s), f(t), \theta) \text { holds. }
\end{aligned}
$$

For 4) we need $\mid$ unify - $\arg (1, f(s), f(t))|>|$ unify $(s, t) \mid$, which holds because $2>1$.

We prove now that $I$ is a $\Theta$-model of UNIFY. The following definition is useful.

Definition 4.7. Let $I$ be a $\Theta$-interpretation. We say that $I$ is good if for all $\langle A, \theta\rangle \in I$ we have $\operatorname{Ran}(\theta) \subseteq \operatorname{Var}(A)$.

In good interpretations the truth of a conjunct (see Definition 2.7) can be checked, as the condition that $(A, \mathbf{B}, \theta, \sigma)$ is a good tuple is not needed. Indeed this condition holds for atoms defined in the program if the interpretation is good and for built-in atoms it follows by Definition 2.1 and the Good Tuple Lemma 2.5.

Lemma 4.8. $I$ is a $\Theta$-model of UNIFY.

Proof. The condition $\operatorname{Ran}(\theta) \subseteq \operatorname{Var}(s, t)$ that occurs in $I$ implies that $I$ is good.

Consider clauses $\left(u_{1}\right)-\left(u_{4}\right) \cdot\langle s=t, \theta\rangle \in \mathbb{I}=\rrbracket$ iff $\theta=m g u(s, t)$, with $\theta$ relevant. Then $\operatorname{Ran}(\theta) \subseteq \operatorname{Var}(s, t)$ and $\operatorname{Inv}(s, t, \theta)$ hold. This implies that $I$ is a $\Theta$-model of $\left(u_{1}\right)-\left(u_{4}\right)$. $I$ is a $\Theta$-model of $\left(u_{5}\right)$, since the relations unify and term - unify are equivalent w.r.t. $I$. I is a $\Theta$-model of $\left(u a r_{1}\right)$, since the condition $\langle$ unify-args $(n, s, t), \theta\rangle \in I$ does not depend on the value of $n$. $I$ is a $\Theta$-model of $\left(u a r_{2}\right)$, because for an atom $A=$ unify $\operatorname{args}(n, s, t)$ and a variant unify - $\operatorname{args}\left(0, X^{\prime}, Y^{\prime}\right) \leftarrow$ of $\left(u a r_{2}\right)$ s.t. $\theta=m g u(A$, unify $\left.\operatorname{args}\left(0, X^{\prime}, Y^{\prime}\right)\right)$ exists, we have that $n=0$ and $\operatorname{Inv}(s, t, \theta \mid A)$ holds. Consider now the clause $(t u)$. Let $A=$ term - unify $(x, y)$ and let term - unify $\left(X^{\prime}, Y^{\prime}\right) \leftarrow$ functor $\left(X^{\prime}, F^{\prime}, N^{\prime}\right)$, functor $\left(Y^{\prime}, F^{\prime}, N^{\prime}\right)$, unify-args $\left(N^{\prime}, X^{\prime}, Y^{\prime}\right)$ be a variant of $(t u)$ disjoint with $A$. Suppose $\alpha=m g u\left(A\right.$, term - unify $\left.\left(X^{\prime}, Y^{\prime}\right)\right)$ exists 
and assume

$$
I \models\left\langle\left(\text { functor }\left(X^{\prime}, F^{\prime}, N^{\prime}\right), \text { functor }\left(Y^{\prime}, F^{\prime}, N^{\prime}\right), \text { unify }-\operatorname{args}\left(N^{\prime}, X^{\prime}, Y^{\prime}\right)\right) \alpha, \eta\right\rangle .
$$

We need to show that $I=\langle A,(\alpha \eta) \mid A\rangle$.

Since $F^{\prime} \alpha$ and $N^{\prime} \alpha$ are in $V a r$, then by the semantics of functor we have that (23) implies that $N^{\prime} \alpha \theta=a(x), \quad F^{\prime} \alpha \theta=f u n c t(x)=f u n c t(y), I \models\left\langle u n i f y-\operatorname{args}\left(N^{\prime}, X^{\prime}, Y^{\prime}\right) \alpha \theta, \beta\right\rangle$, $\eta=\theta \beta$ and $(\alpha \eta) \mid A=\beta$. But for compound terms $x$ and $y$ we have that $I=\langle$ term unify $(x, y), \eta\rangle$ iff $I=\langle$ unify - $\operatorname{args}(a(x), x, y), \eta\rangle$. Then $I=\langle$ term - unify $(x, y),(\alpha \eta) \mid A\rangle$. It remains to check that $I$ is a model of $(u a)$. Let $A=u n i f y-\arg (n, x, y)$ and let unify $-\arg \left(N^{\prime}, X^{\prime}, Y^{\prime}\right) \leftarrow \arg \left(N^{\prime}, X^{\prime}, \operatorname{Arg} X^{\prime}\right), \arg \left(N^{\prime}, Y^{\prime}, \operatorname{Arg} Y^{\prime}\right), \operatorname{unify}\left(\operatorname{Arg} X^{\prime}, \arg Y^{\prime}\right)$ be a variant of $(u a)$ disjoint with $A$. Suppose $\alpha=m g u\left(A, u n i f y-\arg \left(N^{\prime}, X^{\prime}, Y^{\prime}\right)\right)$ exists and assume

$$
I \models\left\langle\left(\arg \left(N^{\prime}, X^{\prime}, \operatorname{Arg} X^{\prime}\right), \arg \left(N^{\prime}, Y^{\prime}, \operatorname{Arg} Y^{\prime}\right), \operatorname{unify}\left(\operatorname{Arg} X^{\prime}, \arg Y^{\prime}\right)\right) \alpha, \eta\right\rangle .
$$

We need to show that $I \models\langle A,(\alpha \eta) \mid A\rangle$. Since $\operatorname{Arg} X^{\prime} \alpha$ and $\operatorname{Arg} Y^{\prime} \alpha$ are in $\operatorname{Var}$, then by the semantics of $\arg$ we have that (24) implies that $N^{\prime} \alpha=n$, with $n>0, \operatorname{Arg} X^{\prime} \alpha \theta=x_{n}$, $\operatorname{Arg} Y^{\prime} \alpha \theta=y_{n}, I=\left\langle\operatorname{unify}\left(\operatorname{Arg} X^{\prime}, \arg Y^{\prime}\right) \alpha \theta, \beta\right\rangle, \eta=\theta \beta$ and $(\alpha \eta) \mid A=\beta$. Now notice that $\operatorname{Dom}(\beta) \subseteq \operatorname{Var}\left(x_{n}, y_{n}\right)$ and $\operatorname{Inv}\left(x_{n} \alpha, y_{n} \alpha, \beta\right)$ imply $\operatorname{Inv}(x \alpha, y \alpha, \beta)$. Then $I \models\langle$ unify $\arg (x, y),(\alpha \eta)|A\rangle$.

This concludes the proof that $I$ is a $\Theta$-model of UNIFY.

We can now prove the desired result.

Theorem 4.9. UNIFY is acceptable w.r.t. $\mid$ and I.

Proof. Notice that any atom in the body of an instance of a clause in UNIFY satisfies property (ii) of Definition 3.13, since each clause with nonempty body is in homogeneous form. Any instance of $\left(u_{1}\right),\left(u_{2}\right),\left(u_{3}\right),\left(u_{4}\right)$ satisfies the appropriate requirement since $\mid$ unify $(s, t)|\rangle\langle 0,0,0\rangle$. Consider now a head instance $C=A \leftarrow B_{1}$, $B_{2}, B_{3}, B_{4}, B_{5}$. of $\left(u_{5}\right)$. $C$ is of the form unify $(s, t) \leftarrow$ nonvar $(s)$, nonvar $(t)$, compound $(s)$, compound $(y)$, term - unify $(s, t)$. We prove two claims which obviously imply that $C$ satisfies the appropriate requirement.

Claim 1. $|A|>\left|B_{i}\right|$ for $i=1, \ldots, 4$.

Proof. Note that $|A|\rangle\langle 0,0,0\rangle=\left|B_{i}\right|$ for $i=1, \ldots, 4$.

Claim 2. Suppose that $I \models\left\langle B_{1}, B_{2}, B_{3}, B_{4}, \eta\right\rangle$. Then $\left.|A|\right\rangle\left|B_{5} \eta\right|$.

Proof. By the semantics of the built-in's nonvar and compound it follows that $s \eta=s$, $t \eta=t$. So $\mid$ unify $(s, t)|=\langle\operatorname{card}(\operatorname{Var}(s, t)), \operatorname{nodes}(s)+\operatorname{nodes}(t), 1\rangle\rangle\langle\operatorname{card}(\operatorname{Var}(s, t))$, $\operatorname{nodes}(s)+\operatorname{nodes}(t), 0\rangle=\mid$ term - unify $(s, t) \mid$.

Consider a head instance $C=A \leftarrow B_{1}, B_{2}, B_{3}$. of $(t u)$. $C$ is of the form

$$
\text { term - unify }(s, t) \leftarrow \operatorname{functor}(s, F, N) \text {, functor }(t, F, N) \text {, unify - } \operatorname{args}(N, s, t) \text {. }
$$

We prove two claims which obviously imply that $C$ satisfies the appropriate requirement.

Claim 1. $|A|>\left|B_{i}\right|$ for $i=1,2$.

Proof. Note that $|A|\rangle\langle 0,0,0\rangle=\left|B_{i}\right|$ for $i=1,2$. 
Claim 2. Suppose that $I \models\left\langle B_{1}, B_{2}, \eta\right\rangle$. Then $\left.|A|\right\rangle\left|B_{3} \eta\right|$.

Proof. By assumption $s \eta=s, t \eta=t$ and $n=a(s)=a(t)$. Notice that nodes $(s)+$ nodes $(t)>f(n, s, t)$. So $\mid$ term - unify $(s, t)|>|$ unify $-\operatorname{args}(n, s, t) \mid$.

Consider a head instance $C=A \leftarrow B_{1}, B_{2}, B_{3}, B_{4}$. of $\left(u a r_{1}\right)$. $C$ is of the form

$$
\text { unify }-\operatorname{args}(n, s, t) \leftarrow n>0, \text { unify }-\arg (n ; s, t), N 1:=n-1 \text {, unify }-\operatorname{args}(N 1, s, t) \text {. }
$$

We prove three claims which obviously imply that $C$ satisfies the appropriate requirement.

Claim 1. $|A|>\left|B_{i}\right|$ for $i=1,3$.

Proof. Note that $|A|\rangle\langle 0,0,0\rangle=\left|B_{i}\right|$ for $i=1,3$

Claim 2. Suppose that $I \models\left\langle B_{1}, \eta\right\rangle$. Then $\left.|A|\right\rangle\left|B_{2} \eta\right|$.

Proof. By the semantics of the built-in $>$ it follows that $s \eta=s, t \eta=t, n>0$. Notice that $f(n, s, t) \geqq \operatorname{nodes}\left(s_{n}\right)+\operatorname{nodes}\left(t_{n}\right)$. So $\mid$ unify $-\operatorname{args}(n, s, t)|>| \operatorname{unify}-\arg (n, s, t) \mid$.

Claim 3. Suppose that $I \models\left\langle B_{1}, B_{2}, B_{3}, \eta\right\rangle$. Then $\left.|A|\right\rangle\left|B_{4} \eta\right|$.

Proof. By the semantics of the built-in's $>,:=$ and of the relation unify-arg it follows that $n>0, a(s) \geqq n>0, N 1 \eta=n-1, \operatorname{Var}(s \eta, t \eta) \leqq \operatorname{Var}(s, t)$. If $\operatorname{Var}(s \eta, t \eta) \subset$ $\operatorname{Var}(s, t)$ then $\operatorname{card}(\operatorname{Var}(s, t))>\operatorname{card}(\operatorname{Var}(s \eta, t \eta))$; if $\operatorname{Var}(s \eta, t \eta)=\operatorname{Var}(s, t)$ then $\operatorname{nodes}(s \eta)+\operatorname{nodes}(t \eta)=\operatorname{nodes}(s)+\operatorname{nodes}(t)$, hence $f(n, s, t)>f(n-1, s \eta, t \eta)$. So in both cases we have $\mid$ unify - $\operatorname{args}(n, s, t)|>|$ unify - $\operatorname{args}(n-1, s \eta, t \eta) \mid$.

Consider a head instance $C=A \leftarrow B_{1}, B_{2}, B_{3}$ of $(u a)$. $C$ is of the form

$$
\text { unify }-\arg (n, s, t) \leftarrow \arg (n, s, \operatorname{Arg} X), \arg (n, t, \operatorname{Arg} Y), \operatorname{unify}(\operatorname{Arg} X, \operatorname{Arg} Y) \text {. }
$$

We prove two claims which obviously imply that $C$ satisfies the approximate requirement.

Claim 1. $|A|>\left|B_{i}\right|$ for $i=1,2$.

Proof. Note that $|A|\rangle\langle 0,0,0\rangle=\left|B_{i}\right|$ for $i=1,2$.

Claim 2. Suppose that $I \models\left\langle B_{1}, B_{2}, \eta\right\rangle$, Then $\left.|A|\right\rangle\left|B_{3} \eta\right|$.

Proof. Since in the clause $C$ the third argument of arg is a fresh variable, then from the semantics of $\arg$ it follows $s \eta=s, t \eta=t, n>0, a(s) \geqq n>0, \operatorname{Arg} X \eta=s_{n}, \operatorname{Arg} Y \eta=$ $t_{n}$. So $\mid$ unify $-\arg (n, s, t)|>|$ unify $\left(s_{n}, t_{n}\right) \mid$.

Consider now the program UNIFYoc for the unification with occur check (see Sterling and Shapiro [page 152] [17]). Let UNIFY' be the program obtained by UNIFY introducing the atom not-occurs-in $(X, Y)$ before the last atom in the bodies of clauses $\left(u_{2}\right)$ and $\left(u_{3}\right)$. Then UNIFYOC is the union of UNIFY' with the following program not-occur defining the relation not-occurs-in/2:

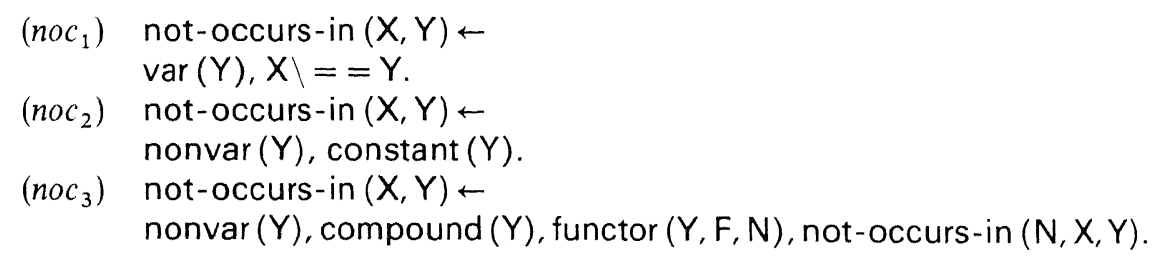




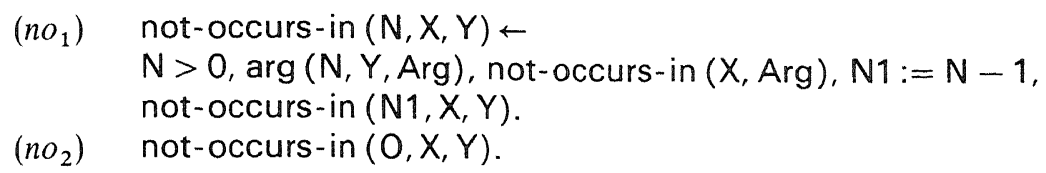

By the Modularity Theorem 4.6 and the Soundness IV Theorem 3.5 to prove that UNIFYoc strongly terminates it suffices to prove that non-occur is acceptable and then prove that UNIFY' is acceptable w.r.t. a $\Theta$-model of UNIFYoc and a level mapping || such that $\mid$ not - occurs $(s, t) \mid=0$ for all $s, t$. with

To prove that not-occur is acceptable we define an appropriate level mapping

$$
\begin{aligned}
& \mid \text { not }- \text { occurs }-\operatorname{in}(x, y) \mid=\operatorname{nodes}(y)+1, \\
& \mid \text { not }- \text { occurs }-\operatorname{in}(n, x, y) \mid=\operatorname{nodes}(y)-\sum_{i=n+1}^{a(y)} \operatorname{nodes}\left(y_{i}\right) \text { if } n>0, \\
& \mid \text { not }- \text { occurs }-\operatorname{in}(0, x, y) \mid=0, \\
& |A|=0 \text { if } A \text { is a built-in. }
\end{aligned}
$$

Next, we define a $\Theta$-interpretation of not-occur by putting

$$
I^{\prime}=\{\langle\text { not }- \text { occurs }- \text { in }(s, t), \varepsilon\rangle\} \cup\{\langle\text { not }- \text { occurs }- \text { in }(n, s, t), \varepsilon\rangle \mid 0 \leqq n \leqq a(t)\} .
$$

Lemma 4.10. $I^{\prime}$ is a $\Theta$-model of not-occur.

Proof. Notice that $1 \leqq n-1 \leqq a(t)$ implies $0 \leqq n \leqq a(t)$. This implies that $I^{\prime}$ is a $\Theta$-model of not-occur.

Lemma 4.11. not-occur is acceptable w.r.t. $\mid$ | and $I^{\prime}$.

Proof. Notice that condition (ii) of Definition 3.4 is satisfied since not-occur is stable. Any instance of $\left(n o c_{1}\right)$ and $\left(n o c_{2}\right)$ satisfies the appropriate requirement since $\mid$ not - occurs - in $(s, t) \mid>0$. Consider an instance $C=A \leftarrow B_{1}, B_{2}, B_{3}, B_{4}$ of $\left(n o c_{3}\right)$. $C$ is of the form not-occurs - in $(s, t) \leftarrow \operatorname{nonvar}(t)$, compound $(t)$, functor $(t, F, N)$, not-occurs - in $(N, s, t)$. We prove two claims which obviously imply that $C$ satisfies the appropriate requirement.

Claim 1. $|A|>\left|B_{i}\right|$ for $i=1,2,3$.

Proof. Notice that $|A|>0=\left|B_{i}\right|$ for $i=1,2,3$.

Claim 2. Suppose that $I^{\prime} \models\left\langle B_{1}, B_{2}, B_{3}, \eta\right\rangle$. Then $\left.|A|\right\rangle\left|B_{4} \eta\right|$.

Proof. By the semantics of the built-in's nonvar, compound and functor we have $s \eta=s, t \eta=t$ and $N \eta=a(t)$. So $\mid$ not - occurs $-i n(s, t) \mid=\operatorname{nodes}(t)+1>\operatorname{nodes}(t)=$ $\mid$ not - occurs $-\operatorname{in}(\mathrm{N \eta}, \mathrm{s}, t) \mid$.

Consider now an instance $C=A \leftarrow B_{1}, B_{2}, B_{3}, B_{4}, B_{5}$ of $\left(n o_{1}\right)$. $C$ is of the form $n o t-\operatorname{occurs}-\operatorname{in}(n, s, t) \leftarrow n>0, \arg (n, t, \operatorname{Arg})$, not $-\operatorname{occurs}-\operatorname{in}(s, \operatorname{Arg}), N 1:=n-1$, not-occurs - in $(N 1, s, t)$.

We prove three claims which obviously imply that $C$ satisfies the appropriate requirement.

Claim 1. $|A|>\left|B_{i}\right|$ for $i=1,2,4$.

Proof. Notice that $|A|>0=\left|B_{i}\right|$ for $i=1,2,4$. 
Claim 2. Suppose that $I^{\prime} \models\left\langle B_{1}, B_{2}, \eta\right\rangle$. Then $\left.|A|\right\rangle\left|B_{3} \eta\right|$.

Proof. By the semantics of the built-in's $>$ and $\arg$ we have $s \eta=s, t \eta=t, \operatorname{Arg} \eta=t_{n}$ and $1 \leqq n \leqq a(t)$. So $\leqslant n o t-\operatorname{occurs}-\operatorname{in}(n, s, t) \mid=\operatorname{nodes}(t)-\sum_{i=n+1}^{a(t)} \operatorname{nodes}\left(t_{i}\right)>$ $\operatorname{nodes}\left(t_{n}\right)+1=\mid$ not - occurs - in $\left(s, t_{n}\right) \mid$.

Claim 3. Suppose that $I^{\prime} \models\left\langle B_{1}, B_{2}, B_{3}, B_{4}, \eta\right\rangle$. Then $\left.|A|\right\rangle\left|B_{5} \eta\right|$.

Proof. By the semantics of the built-in's $>, \arg ,:=$ and of the relation not - occurs in we have $s \eta=s, t \eta=t, \operatorname{Arg} \eta=t_{n}, N 1 \eta=n-1(\geqq 0)$ and $(1 \leqq n \leqq a(t))$. So $\mid$ not occurs $-i n(n, s, t)\left|=\operatorname{nodes}(y)-\sum_{i=n+1}^{a(t)} \operatorname{nodes}\left(t_{i}\right)>\operatorname{nodes}(t)-\sum_{i=n-1}^{a(t)} \operatorname{nodes}\left(t_{i}\right)=\right|$ not occurs - in $(n-1, s, t) \mid$.

To prove that UNIFY' is acceptable we consider the level mapping and $\Theta$-model defined in UNIFY and we treat not-occurs-in as built-in relation whose semantics is given by $I^{\prime}$.

Lemma 4.12. UNIFY' is acceptable w.r.t. | | and I.

Proof. Notice that if $C=A \leftarrow B_{1}, \ldots, B_{4}$ is an instance of $\left(u_{2}^{\prime}\right)$ (resp. of $\left(u_{3}^{\prime}\right)$ exchanging the positions of $s$ and $t$ in the body of the clause), then $C$ is of the form

unify $(s, t) \leftarrow \operatorname{var}(s)$, nonvar $(t)$, not - occurs - in $(s, t), s=t$.

If $I=\left\langle B_{1}, B_{2}, B_{3}, \eta\right\rangle$ then by the semantics of the built-in's var, nonvar and of the relation not-occurs - in we have $s \eta=s$ and $t \eta=t$, i.e. not-occurs-in $(s, t)$ does not modify $s$ and $t$. It follows that the proof that UNIFY' is acceptable w.r.t. || and $I$ is analogous to the one for UNIFY given in Theorem 4.9.

Acknowledgements. We thank Annalisa Bossi and Kees Doets for helpful discussions on the subject of the Good Tuple Lemma 2.5. Also, we thank the referees for useful suggestions on the subject of this paper.

Notes. This research was partly done during the third author's stay at Centre for Mathematics and Computer Science, Amsterdam. The work of K.R. Apt and E. Marchiori was partly supported by ESPRIT Basic Research Action 6810 (Compulog 2). The work of C. Palamidessi was partly supported by ESPRIT Basic Research Action 3020 (Integration) and by the Italian CNR (Consiglio Nazionale delle Ricerche). The work of E. Marchiori was also partly supported by the Italian CNR under Grant No. 89.00026.69.

\section{References}

1. Apt, K. R.: Logic Programming. In: van Leeuwen, J. (ed.) Handbook of Theoretical Computer Science. Vol. B. Elsevier 1990

2. Apt, K. R., Bezem, M.: Acyclic Programs. New Generation Comput. 9, 335-363 (1991)

3. Apt, K. R., Doets, H. C.: A new definition of SLDNF-resolution. Tech. Rep. CS-R9242. CWI, Amsterdam, NL (1992)

4. Apt, K. R., Marchiori, E., Palamidessi, C.: A declarative approach for first-order built-in's of Prolog. Tech. Rep. CS-R9246. CWI, Amsterdam, NL (1992)

5. Apt, K. R., Pedreschi, D.: Studies in pure Prolog: termination. In: Lloyd, J. W. (ed.) Symposium on Compuational Logic. Berlin, Heidelberg, New York: Springer (1990)

6. Bezem, M.: Characterizing termination of logic programs with level mappings. In: Lusk, E. L., Overbeek, R. A. (eds.) Proceedings of the North American Conference on Logic Programming, 69-80. The MIT Press (1989) 
7. Börger, E.: A logical operational semantics of full Prolog, Part III: Built-in predicates for files, terms, arithmetic and input-output. In: Moschovakis Y. (ed.), Proceedings Workshop on Logic from Computer Science. Berlin, Heidelberg, New York: Springer MSRI Publications (1989)

8. Bossi, A., Cocco, N., Fabris, M.: Proving termination of logic programs by exploiting term properties. In: Proceedings of Tapsoft '91, pp. 153-180 (1991)

9. Cavedon, L.: Continuity, consistency, and completeness properties for logic programs. In: Levi, G., Martelli, M. (eds.) Proceedings of the Sixth International Conference on Logic Programming, pp. 571-584. The MIT Press (1989)

10. Clark, K. L.: Predicate logic as a computational formalism. Tech. Rep. DOC 79/59. ico, London, GB (1979)

11. Deransart, P., Ferrand, G.: An operational formal definition of Prolog. In: Proceedings of the 4th Symposium on Logic Programming, pp. 162-172. Computer Society Press (1987)

12. Falaschi, M., Levi, G., Martelli, M., Palamidessi, C.: Declarative modeling of the operational behaviour of logic languages. Theoret. Comput. Sci. 69, 289-318 (1989)

13. Hill, P. M., Lloyd, J. W.: Analysis of meta-programs. In: Abramson, H. D., Rogers, M. H. (eds.) Proceedings of the Meta88 Workshop, pp. 23-52. MIT Press (1988)

14. Lloyd, J. W.: Foundations of Logic Programming. Second ed., Berlin, Heidelberg, New York: Springer 1987

15. Pieramico, C.: Metodi formali di ragionamento sulla terminazione di programmi prolog. Tech. Rep. Tesi di Laurea. Universitá degli Studi di Pisa. I (1991)

16. Plümer, L.: Automatic termination proofs for prolog programs operating on nonground terms. In: Proceedings of the 1991 International Logic Programming Symposium. San Diego (1991)

17. Sterling, L., Shapiro, E.: The Art of Prolog. MIT Press 1986

18. van Emden, M. H., Kowalski, R. A.: The semantics of predicate logic as a programming language. Journal of the ACM 23, 733-742 (1976) 